Review

\title{
Magnetically Separable and Sustainable Nanostructured Catalysts for Heterogeneous Reduction of Nitroaromatics
}

\section{Mohammadreza Shokouhimehr}

School of Chemical and Biological Engineering, Seoul National University, Seoul 151-744, Korea; E-Mail: mrsh2@snu.ac.kr; Tel.: +82-10-5842-9632

Academic Editor: Masahiko Arai

Received: 28 December 2014 / Accepted: 5 March 2015 / Published: 31 March 2015

\begin{abstract}
This review is focused on the strategies and designs of magnetic nanostructured catalysts showing the enhanced and sustainable catalytic performances for the heterogeneous reduction of nitoaromatics. Magnetic catalysts have the benefits of easy recovery and reuse after the completion of the reactions and green chemical processes. Magnetic separation, among the various procedures for removing catalysts, not only obviates the requirement of catalyst filtration or centrifugation after the completion of reactions, but also provides a practical technique for recycling the magnetized nanostructured catalysts. Consequently, discussions will address the methodologies and exemplars for the reusable magnetic composite catalysts. Because the synthesis of ideal magnetic nanostructured catalysts is of primary importance in the development of high-quality sustainable processes, the designs, preparation methods and recyclability of various recoverable magnetic nanostructured catalysts are emphasized. The representative methods and strategies for the synthesis of durable and reusable magnetic nanostructured catalysts are highlighted. The advantages, disadvantages, recyclability and the efficiency of the introduced heterogeneous systems have been explored in the reduction of nitrobenzene derivatives.
\end{abstract}

Keywords: reduction; nitroaromatic; nanostructure; heterogeneous catalysis; nanocatalyst; hydrogenation; catalyst; nitrobenzene; magnetic recycling

\section{Introduction}

The hydrogenation of organic compounds is a fundamental chemical transformation for the production of a large variety of important intermediates in the laboratory and industry [1,2]. 
The reduction of nitroarenes to anilines is widely used in chemical processes, because aromatic amines are highly important chemicals for the preparation of fine chemical products, such as agrochemicals, pharmaceuticals, polymers, dyes, pesticides and cosmetics [3]. For example, the majority of the globally consumed aniline is produced by catalytic routs, which is used for the synthesis of methylene diphenyl diisocyanate and rubber processing [4]. The mechanism for the hydrogenation of nitrobenzene was proposed by Haber in 1898 [5]. The transformation to aniline occurs in a three-step process involving nitrosobenzene and phenylhydroxylamine (Scheme 1). The nitro group is first reduced to a nitroso group, and then, a second equivalent of hydrogen can result in the formation of hydroxylamine. Finally, the aniline is obtained with the addition of a third equivalent hydrogen. It was also proposed that azoxybenzene, azobenzene and hydrazobenzene by-products could be formed via the reaction of two intermediate species.

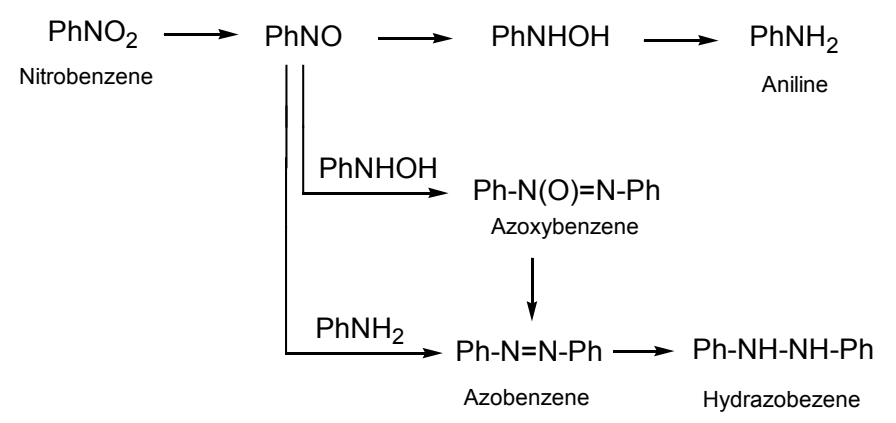

Scheme 1. The Haber mechanism for the hydrogenation of nitrobenzene to aniline.

Although significant progress has been achieved to improve the substrate breadth, there are still major problems of applied catalysts in reduction processes. Commercially, the reduction of nitroaromatics carried out in the gas phase using a nickel, copper or iron catalyst in the presence of various proton sources produces a large amount of wastes, which are environmentally undesirable [6]. In addition, high temperature and hydrogen pressure are other restrictions associated with these catalytic processes [7]. The classical methods for the hydrogenation of nitro compounds use various homogeneous transition-metal catalysts, e.g., Pd, Pt, Ru, etc. [8-10]. Homogenous catalysts generally dissolve in reaction media, thus providing more readily-accessible active catalytic sites, which results in mild reaction conditions and good selectivity. These catalysts, however, have limited usages due to the problems in separating the products contaminated with unstable residuals and recycling the expensive catalysts [11]. The removal of residual catalytic species from the reaction mixture is always challenging, even with the use of numerous techniques, such as chromatography, distillation or extraction. In contrast, heterogeneous catalysts have the advantages of recovery and reuse after the completion of the reactions and green chemical processes [12-14]. Therefore, several procedures have been developed to synthesize durable and reusable heterogeneous catalysts for the reduction of nitro compounds. These catalysts are indeed very favorable from a sustainable point of view [15]. In the past decade, many efforts have been dedicated for the immobilization of metal complexes on various supports to combine the privileges of both homogeneous and heterogeneous catalysts [16,17]. Furthermore, industrial heterogeneous catalysts composed of supported metal particles or NPs were also prepared for practical applications. However, while supporting the catalyst can increase their stability, it decreases their reactivity [18]. Although using these catalysts achieved some important criteria of an ideal catalyst, such 
as reusability and cost effectiveness, significant drawbacks, including low catalytic activity and selectivity, sever reaction conditions, side reaction production and metal species leaching, remained unresolved [19]. Consequently, exploring economical methods to prepare highly reactive, stable, scalable and recyclable heterogeneous catalysts remains as an important issue in the catalysis research field. Recent developments and applications of nanoscience and nanotechnology in the field of catalysis could satisfy the needs of such improved catalyst systems. The designed magnetic nanostructured catalysts can substitute typical heterogeneous catalysts and serve as more active sustainable catalysts for various catalytic transformations. Nanostructured catalysts as a bridge between homogeneous and heterogeneous catalysts present high catalytic activities because of their high surface-to-volume ratio. In this review article, recent major achievements on sustainable magnetic nanostructured catalysts are highlighted. Discussions will emphasize the strategies and designs of magnetically separable and reusable nanostructured catalysts, showing enhanced and sustainable catalytic performances, which will lead to the development of efficient and effective catalysts. In addition, explorations will address the methodologies and exemplars for the recyclable magnetic nanostructured catalysts. The prospective applications, advantages and challenges of the introduced heterogeneous systems are studied.

\section{Non-Magnetic Nanostructured Catalysts}

Heterogeneous nanostructured catalysts have the benefits of recovery and reuse after completion of the reactions and green chemical processes. However, each designed synthesized heterogeneous nanostructured catalyst has also shortcomings and disadvantages, which need to be overcome by more developed techniques. For instance, noble metal nanoparticles (NPs) as a pivotal class of nanostructured catalysts possess significant potentials owing to their tunable size, shape-dependent catalytic activity and selectivity. Recent advancements in the synthesis of uniformly-sized NPs offer many opportunities to enhance the catalytic performances [20]. Due to their small sizes, NP-based catalysts (nanocatalysts) have high surface areas, increase the exposed active sites and thereby improve contact areas with reactants. However, the high surface energy of nanocatalysts also leads to the instability in the reaction mixture, resulting in aggregation and loss of catalytic activity. Although nanocatalysts have high catalytic activities because of their high surface-to-volume ratio, they suffer from very critical drawbacks, such as the scale-up process and reusability. In practice, the high surface energy of nanostructures gives rise to their instability in different reaction conditions and aggregation, causing the rapid loss of catalytic activity [21]. Recovering the nanocatalysts also requires a high speed centrifugation step or a tedious workup of the final reaction mixture. Furthermore, it has been shown that nanocatalysts generally do not retain high catalytic activity without a sturdy support. Nanocatalysts without any support are generally unstable, and structural deformation occurs after a single use in catalytic reactions [22-24]. The aggregation of nanocatalysts reduces the active surface area, resulting in less effective chemical reactions.

The fabrication of various metal nanocatalysts supported on nano-sized solid materials has been introduced and investigated to overcome the problems pertaining to the unsupported nanocatalysts [25]. In order to control the catalytic properties and improve the stability of the nanocatalysts, the catalytically-active metal NPs can be immobilized on nano-sized solid supports. The well-dispersed nanocatalysts on mesoporous nanospheres are more stable than unsupported nanocatalysts, providing a 
green and environmentally benign protocol. These nanocatalysts can be separated by a centrifuge and be reused several times [26]. However, these nanostructured catalysts can be generally prepared in a small scale through multistep processes. In addition, the centrifugation step for the recovery and reuse of the catalysts is not a desirable procedure, because of catalyst loss and the residues remaining in each recycle. Such complicated approaches are neither efficient nor operative for the extensive production and applications that are particularly important in chemical industries [27]. These facts have motivated continuous research investigations for the development of highly active and sustainable nanocomposite catalysts.

An efficient, stable and cost-effective synthesis of micro-sized composites as hosts for nanocatalysts is of great importance, as it allows effective utilization and recycling of the expensive metal catalysts. Because the supports strongly affect the activity of nanocatalysts, numerous studies have been performed to improve their properties [28]. The choice of support plays a crucial role in the accessibility to catalytic sites. Of the wide range of catalyst supports, organic polymers represent a very popular commercialized support. Inorganic solid supports, such as metal oxides, carbon, zeolite, zirconia, hydrotalcite and clay, generally show higher thermal and mechanical resistances compared with their organic counterparts. Mesoporous silica and alumina supports are probably the most popular matrixes as inorganic solid supports due to the cost, large surface area and porosity, availability, mechanical robustness and facile synthesis [29-31]. Supporting the highly active nanocatalysts can avoid the problem of recycling and reusability. However, these heterogeneous systems require a filtration step to recover the catalysts. The loss of catalyst, accumulation and sintering during transferring and recycling hinder the efficiency and practicability of these catalysts. Indeed, it is highly essential to combine variable strategies and physiochemical properties of hybrid materials to design and develop superior practical and ideal catalysts [32]. A strategy to circumvent the aforementioned problems is the exploitation and combination of magnetic NPs with the above non-magnetic nanostructure properties to produce a more practical and easier technique for recycling nanocatalysts. The next section explores the strategies and designs of magnetically separable and reusable nanostructured catalysts with the enhanced and sustainable catalytic performances.

\section{Sustainable Magnetic Nanostructured Catalysts}

Magnetic separation techniques have been used for decades in many industries to separate magnetic materials from non-magnetic materials. Numerous opportunities and applications have arisen for hybrid nanomaterial developments by combining magnetic properties. Accordingly, magnetic NPs have attracted great interests owing to their unique characteristics and potential applications in various fields, such as magnetically-assisted drug delivery, magnetic resonance imaging, hyperthermia, magnetic separation of biomolecules, environmental remediation and, especially, the isolation and recycling of expensive catalysts [33]. As shown in Scheme 2, the catalysts incorporated with magnetic species can be easily separated from the reaction mixture. Magnetic separation eliminates the necessity of centrifugation, filtration or other impractical procedures for the recycling of the precious catalysts. It provides a convenient method for isolating and dispersing the magnetized catalysts by applying and removing an appropriate magnetic field. This approach prevents the agglomeration of the catalysts during the reuse and increases their durability. 


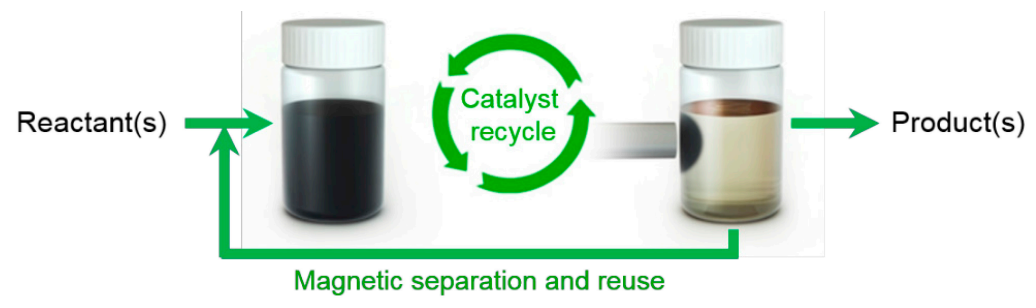

Scheme 2. Magnetic separation and recycling of the magnetized catalysts.

Magnetic NPs can play a dual role in serving both as catalysts and as magnetic carriers for catalysts [34]. Bare magnetic NPs have been successfully used as catalysts in some catalytic processes. They could also be protected with inorganic supports or directly decorated with transition-metal NPs [35]. In the latter usages, magnetic NPs have been extensively employed as alternative catalyst supports. They can be also incorporated as a component in the designed nanostructured and nanocomposite catalysts. The unprotected magnetic NPs are generally stable enough to tolerate most chemical reaction environments. Their exceptional instabilities in extremely acidic, drastic or corrosive reaction conditions, however, can also be resolved by engineering the design and fabrication strategies of the hybrid nanostructured catalysts [36]. To circumvent this drawback, an impermeable dense silica layer has been coated on the core magnetic NPs to protect them from destructive erosion during the harsh reaction conditions [37]. To pursue innovative applications and developments, hybrid magnetic nanocomposites have been designed and applied for many kinds of organic transformations, including reductions. The notable methods and approaches to synthesize efficient magnetic nanostructured catalysts will be the subject of this review article.

\subsection{Magnetic Nanoparticles-Supported Nanocatalysts}

Functionalized magnetic NPs have emerged as robust, viable and readily available alternatives to conventional materials for catalyst support. Magnetic NP-supported catalysts have been efficiently employed as heterogeneous catalysts for various organic transformations [38]. Magnetic NP-supported $\mathrm{Rh}, \mathrm{Pd}$ and Pt catalysts have been widely employed for promoting different organic reactions [39-41]. In most cases, the nanocatalysts are immobilized onto the modified surface of the magnetic NPs. The magnetic NPs of these systems are generally stabilized with suitable capping agents, rendering their stability and dispersibility to organic solvents. The catalysts species can be loaded onto the magnetic supports either by post-modification of magnetic NP functions or by co-precipitation during the magnetic NP synthesis [42]. The use of these magnetic NP catalysts can address the isolation and recycling problem encountered in many catalytic reactions. Most importantly, the magnetic NP-supported catalysts show not only high catalytic activity, but also a high chemical stability.

Gawande et al. synthesized iron oxide NPs and decorated them with nickel nanocatalysts directly on the magnetic NPs by the chemical reduction of nickel chloride. The designed magnetic catalysts found high ragio- and chemo-selectivity for the reduction of nitro compounds under the environmentally-friendly solvent, glycerol, as a hydrogen source, along with a high level of reusability [43]. In another effort, Gawande and colleagues introduced a sustainable protocol for the reduction of aromatic nitro compounds using a magnetically-separable and reusable magnetite supporting gold nanocatalysts under mild reaction conditions [44]. The nanocatalysts were prepared by 
the wet impregnation of a gold precursor on magnetic NPs followed by chemical reduction (Scheme 3). $\mathrm{FeSO}_{4}$ and $\mathrm{FeCl}_{3}$ were dissolved in deionized water under a $\mathrm{N}_{2}$ atmosphere. The resulting mixture was stirred for $15 \mathrm{~min}$ and heated at $60{ }^{\circ} \mathrm{C}$ under vigorous stirring. When the temperature reached $60{ }^{\circ} \mathrm{C}$, an aqueous $\mathrm{NH}_{4} \mathrm{OH}$ solution was added to the mixture. A black precipitate was immediately formed, and heating continued for $2 \mathrm{~h}$. After vigorous stirring, the precipitate was magnetically separated and washed thoroughly with water until the supernatant liquor became neutral. The obtained magnetic NPs were redispersed in water containing gold(III) chloride. The $\mathrm{pH}$ of the solution was adjusted to $12-13$ by adding $\mathrm{NaOH}$ and further stirred for $20 \mathrm{~h}$. The product was washed with water and dried in an oven to yield the magnetic gold nanocatalysts.

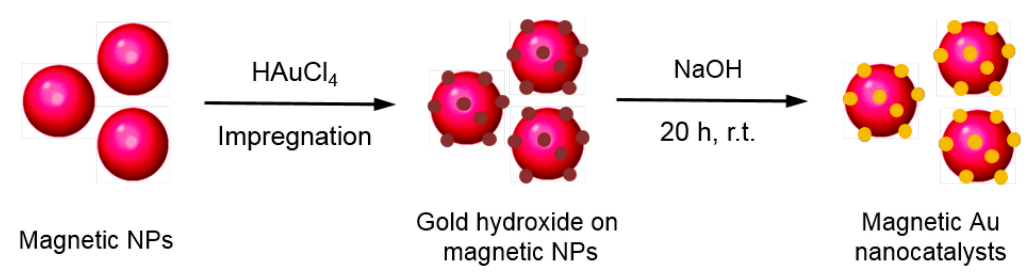

Scheme 3. Synthetic procedure leading to the magnetic Au nanocatalysts.

The magnetic gold nanocatalysts were characterized by several techniques, such as X-ray diffraction (XRD), X-ray photoelectron spectroscopy (XPS), inductively-coupled plasma-atomic emission spectroscopy (ICP-AES), transmission electron microscopy (TEM), field-emission scanning electron microscopy (FE-SEM), energy-dispersive X-ray spectroscopy (EDX) and scanning transmission electron microscopy (STEM). The percentage amount of gold in the magnetic nanocatalysts was determined by ICP-AES, which was in accordance with the XRD results. The TEM image of the catalysts revealed that the overall diameter of the as-synthesized NPs is in the range 20-30 nm. The EDX profile clearly indicated the presence of $\mathrm{Fe}$ and $\mathrm{Au}$ species in the catalysts. It could be clearly seen from the element mapping that the elements $\mathrm{Au}, \mathrm{Fe}$ and $\mathrm{O}$ truly existed in the sample.

The catalytic efficiency of the synthesized magnetic gold nanocatalysts was investigated in the reduction of nitrobenzene as a model substrate. The catalyst showed good catalytic performance in the reduction reaction employing $\mathrm{HCOONH}_{4}$ as a hydrogen source under ambient conditions. Notably, the reduction of nitro compounds does not occur even with a large amount of $\mathrm{HCOONH}_{4}$ in the absence of nanocatalysts. The gold nanocatalysts was ineffective in the absence of $\mathrm{HCOONH}_{4}$ under the same reaction conditions, indicating that ammonium formate is necessary to drive the reduction process. After the optimization of the reaction conditions, the catalytic efficiency of the magnetic gold nanocatalysts was further explored with other substituted nitrobenzene derivatives (Figure 1). It was noted that these reduction reactions with the magnetic nanocatalysts exhibit excellent activity and selectivity with various functional groups.

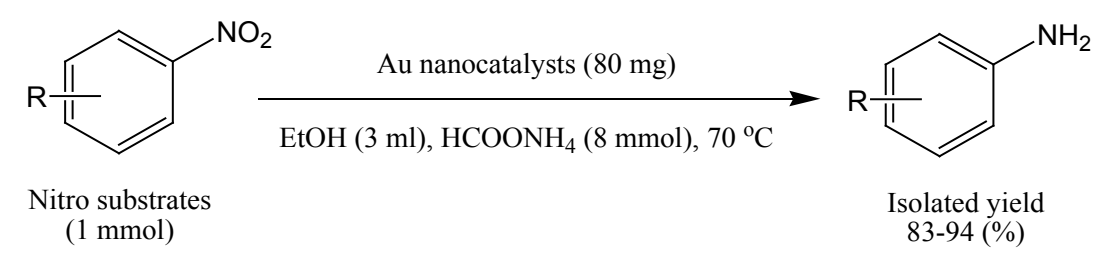

Figure 1. Hydrogenation of nitrobenzene derivatives. 
The stability of the magnetic nanocatalysts was assessed for sequential recyclability in the reduction of nitrobenzene to the corresponding product by magnetic recycling and reuse of the magnetic $\mathrm{Au}$ catalyst under the optimized reaction conditions. It is important to note that a slight loss in the yield of aniline was observed. After the completion of the reaction and between each cycle, the nanocatalysts could be easily separated by a simple magnetic decantation using an external magnet and subjected to the next cycle. It is noteworthy that, although the nanocatalysts stabilized on magnetic NPs offer good catalytic activity in many organic reactions, the modification steps may decrease the magnetization and stability of the magnetic NPs by altering interparticle interactions, which reduce the effective surface area, the catalyst recovery and, hence, the catalytic efficacy (Figure 2). Therefore, catalytic activity decay seems to result from the loss of nanocatalysts during the steps of washing and magnetic separation for the consecutive reuse of the nanocatalysts.

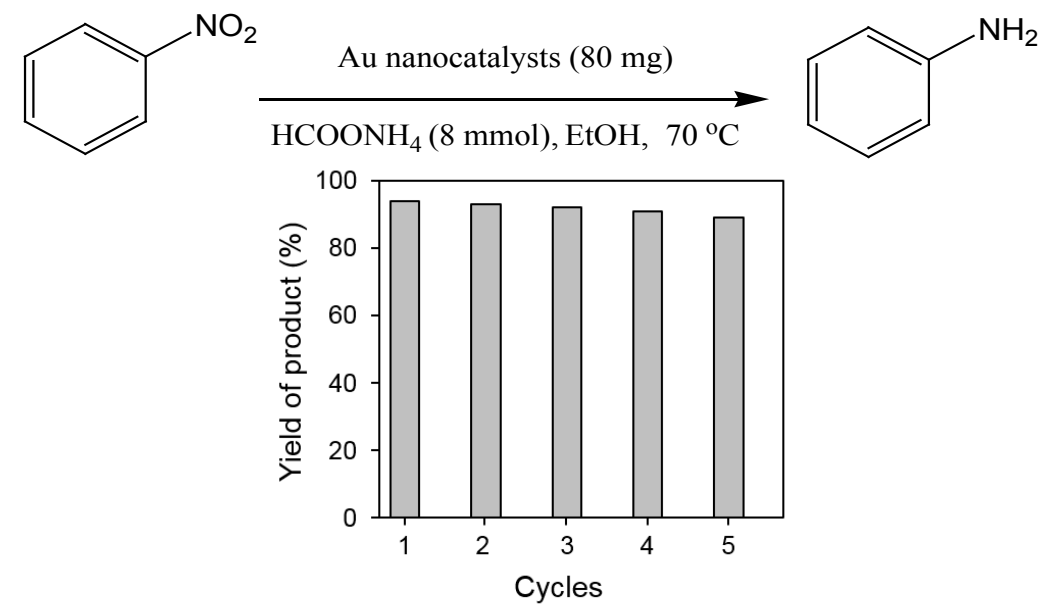

Figure 2. Reusability of the magnetic Au nanocatalysts in the reduction of nitrobenzene.

An easy and straightforward method has been introduced by Pelission et al. for loading palladium NPs onto the magnetic surface of magnetic iron oxide NPs without using organic modifiers [45]. The potential and effectiveness of the $\mathrm{Pd} @ \gamma-\mathrm{Fe}_{2} \mathrm{O}_{3}$ nanocatalysts were investigated in the hydrogenation of nitroarene derivatives under hydrogen pressure $(100 \mathrm{kPa})$ in water. The magnetic palladium nanocatalysts were characterized with a wide range of physicochemical techniques to determine the particle size and morphology, as well as their chemical composition, their crystalline structure and their magnetic behavior. In the TEM images and high-resolution TEM (HRTEM), relatively uniform iron oxide NPs with an average diameter of $11 \mathrm{~nm}$ and Pd NPs of $3 \mathrm{~nm}$ were observed. The magnetization curve of the iron oxide NPs at room temperature showed the characteristic of a superparamagnetic NPs. After characterizations, the magnetically recoverable nanocatalysts were evaluated in a model reaction, such as the reduction of nitroarenes owing to the great potential of the resulting anilines as building blocks in the chemical industry. The reduction of nitroarenes into corresponding anilines was investigated by using the $\mathrm{Pd} @ \gamma-\mathrm{Fe}_{2} \mathrm{O}_{3}$ nanocatalysts under $100 \mathrm{kPa} \mathrm{H}_{2}$ at room temperature in water. No reduction of the nitro groups was observed with the $\gamma-\mathrm{Fe}_{2} \mathrm{O}_{3} \mathrm{NPs}$. However, the $\mathrm{Pd} @ \gamma-\mathrm{Fe}_{2} \mathrm{O}_{3}$ nanocatalysts were active for the hydrogenation of nitrobenzene with a TOF up to $150 \mathrm{~h}^{-1}$. The catalytic lifetime of the magnetic Pd nanocatalysts was studied for the successive hydrogenations of nitrobenzene under hydrogen pressure. The magnetic nanocatalysts were easily 
separated from the reaction mixture by using an external neodymium magnet and washed with water before the reuse under the same conditions. The results for five runs are shown in Table 1. The catalyst could be efficiently reused for five successive runs without loss of catalytic activity.

Table 1. Magnetic recovery and recycling of the $\mathrm{Pd} @ \gamma-\mathrm{Fe}_{2} \mathrm{O}_{3}$ nanocatalysts in the hydrogenation of nitrobenzene.

\begin{tabular}{crrrrr} 
& & & \\
\hline Successive cycle & 1st & 2nd & 3rd & 4th & 5th \\
\hline $\mathrm{TOF} / \mathrm{h}^{-1}$ & 150 & 150 & 150 & 150 & 150 \\
\hline
\end{tabular}

\subsection{Nano-Sized Magnetic and Porous Nanocomposite Catalysts}

The fabrication of various metal nanocatalysts supported on nano-sized solid materials has attracted considerable attention to resolve the problems pertaining to the unsupported nanocatalysts and environmental issues [46]. In order to control the catalytic properties and improve the stability of nanocatalysts, the catalytically-active metal NPs can be immobilized on nano-sized solid supports. Wang et al. developed highly efficient heterogeneous catalysts consisting of silica nanospheres decorated with Pd NPs encapsulated in a nanoporous silica shell. The designed heterogeneous catalyst exhibited high catalytic activity and turn over numbers in the hydrogenation reaction of various substrates, including nitrobenzene at room temperature and 20 bar hydrogen pressure [47]. Among the various nano-sized solid supports, mesoporous silica nanospheres (KCC-1) are particularly important supports for heterogeneous catalysis due to their excellent stability, high surface area, tunable pore size and chemical inertness [48]. Inspired by the above mentioned properties, Dong et al. immobilized Ag NPs on KCC-1 to increase the stability of the Ag NPs and provide highly effective nanocatalysts for the catalytic reduction of nitroaromatic compounds, leading to the safe and eco-friendly reaction conditions [49]. Ag NPs with an average diameter of about $4 \mathrm{~nm}$ were well-dispersed on the fibers of the KCC-1 nanospheres without aggregation and showed excellent catalytic activity for the reduction of 4-nitrophenol and 2-nitroaniline, owing to the easy accessibility of the active sites. The nanocatalysts could be separated by a centrifuge and be reused several times. However, the centrifugation step for the recovery and reuse of the catalysts is not a desirable procedure, because of catalyst loss and the remaining residues in each recycling. Such complicated approaches are neither efficient nor operative for the extensive production and applications that are particularly important in chemical industries [50]. These facts motivated continuous research improvements by the same group for the developments of highly active and magnetically recyclable $\mathrm{Ni@Pd} / \mathrm{KCC}-1$ nanocatalyst [51]. The immobilization of $\mathrm{Ni}$ @Pd NPs on the KCC-1 to fabricate the magnetic recyclable nanocatalysts could be promising for the purpose of preventing the aggregation of nanocatalysts, increasing the accessibility of the active sites and conferring the magnetic separation property to the nanocatalysts. The designed $\mathrm{Ni}$ @Pd/KCC-1 nanocatalysts were employed as highly efficient and reactive catalyst for the reduction of 4-nitrophenol and 4-chlorophenol under ecofriendly conditions. Importantly, the Ni cores of the $\mathrm{Ni}$ @Pd NPs reduced the consumption of Pd and simultaneously made the catalyst reusable, due to its convenient magnetic recovery. The schematic describing the preparation of $\mathrm{Ni} @ \mathrm{Pd} / \mathrm{KCC}-1$ 
nanocatalysts is shown in Scheme 4. Ni@Pd NPs were first synthesized via a one-pot high-temperature solution phase synthesis, including the consecutive reduction of nickel (II) and palladium (II), according to a reported procedure [52]. KCC-1 was prepared by a hydrothermal method and functionalized with 3-mercaptopropyltriethoxysilane. Finally, $0.40 \mathrm{~g}$ of the functionalized KCC-1 was ultrasonically dispersed in $\mathrm{H}_{2} \mathrm{O}$, and $0.08 \mathrm{~g}$ of Ni@Pd NPs was added. After being stirred and ultrasonically dispersed for 1 h, the magnetically recoverable $\mathrm{Ni} @ \mathrm{Pd} / \mathrm{KCC}-1$ nanocatalysts were obtained and dried in a vacuum.

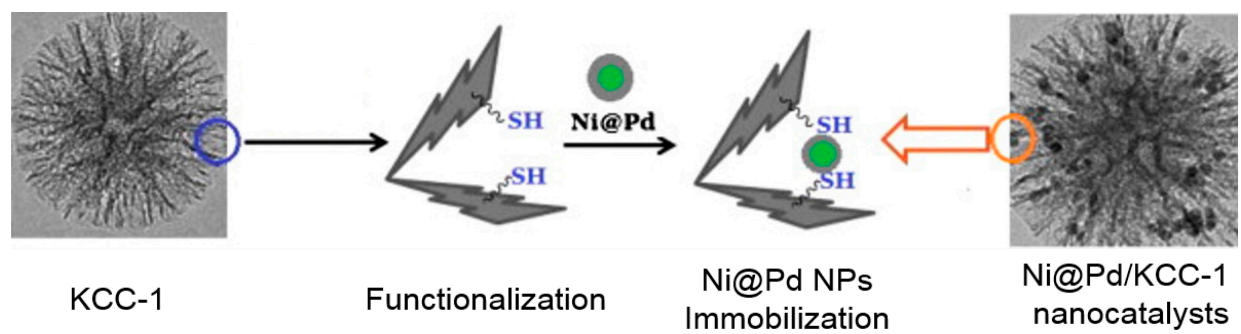

Scheme 4. Synthesis of magnetic Ni@Pd/KCC-1 nanocatalysts. Reprinted with permission from [51].

Figure 3 presents the TEM and HRTEM images of the KCC-1 and Ni@Pd/KCC-1 nanocatalysts with a relatively uniform spherical shape. The Ni@Pd NPs have a narrow size distribution with a mean particle size of $\sim 10 \mathrm{~nm}$. The TEM image of the synthesized KCC-1 mesoporous silica shows the average diameter of 200-300 $\mathrm{nm}$. The HRTEM image shown in Figure $3 \mathrm{~b}$ further clarifies that the distance between the two fibers is $\sim 20 \mathrm{~nm}$. The large distance between the fibers and the high surface area of KCC-1 leads to the easy loading of Ni@Pd NPs. The HRTEM image of the Ni@Pd/KCC-1, shown in Figure 3d, clearly shows well dispersion of nanocatalysts in mesoporous silica.

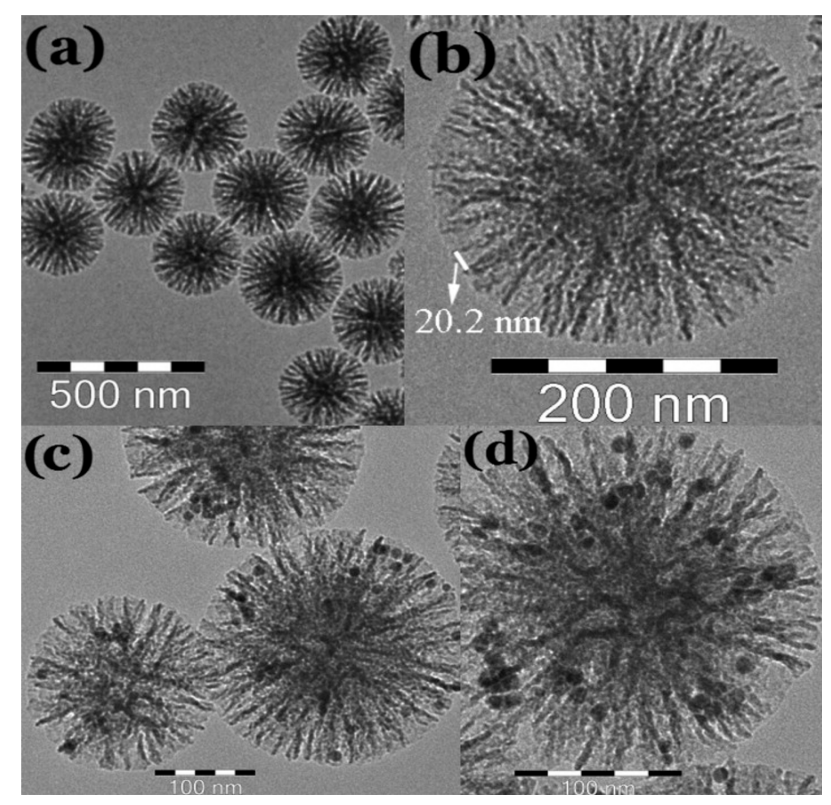

Figure 3. (a) TEM and (b) HRTEM images of KCC-1; (c) TEM and (d) HRTEM images of magnetic Ni@Pd/KCC-1 nanocatalysts. Reprinted with permission from [51].

The catalytic activity of the $\mathrm{Ni@Pd/KCC}-1$ nanocatalysts was verified by the reduction of 4-nitrophenol to 4-aminophenol using $\mathrm{NaBH}_{4}$ as the reductant. The evolution process for reaction time 
was monitored by UV-VIS spectroscopy. The solution of 4-nitrophenol exhibits a strong absorption peak at $317 \mathrm{~nm}$, which is remarkably red-shifted to $400 \mathrm{~nm}$ when treated with an aqueous solution of $\mathrm{NaBH}_{4}$, due to the formation of 4-nitrophenolate ions (Figure 4a). The addition of $\mathrm{Ni} @ \mathrm{Pd} / \mathrm{KCC}-1$ nanocatalysts $(0.4 \mathrm{mg})$ into the system led to the decrease in the peak intensity of 4-nitrophenolateion at $400 \mathrm{~nm}$ with the concomitant increase in the peaks corresponding to 4 -aminophenol at $300 \mathrm{~nm}$. The reaction proceeded rapidly, with the conversion exceeding $99.8 \%$ at a reaction time of about 4 min (Figure $4 b$ ).
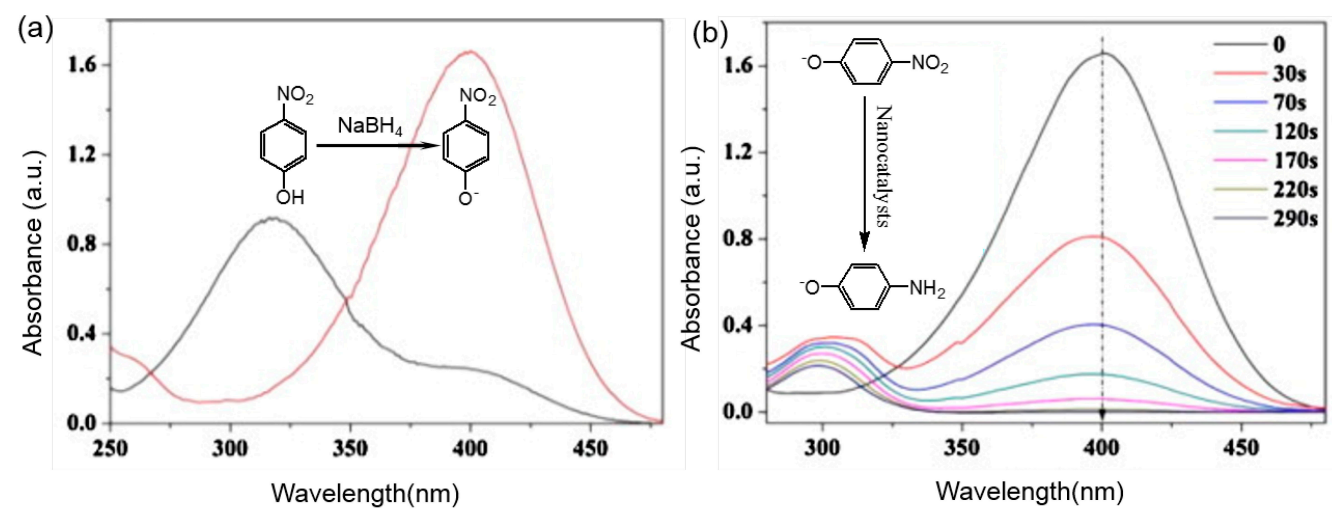

Figure 4. (a) UV-VIS spectra of 4-nitrophenol before and after adding $\mathrm{NaBH}_{4}$ solution; (b) the reduction of 4-nitrophenol over the magneticaaly recyclable $\mathrm{Ni@Pd/KCC}-1$ nanocatalysts. Reprinted with permission from [51].

Moreover, the comparison between the $\mathrm{Ni} @ \mathrm{Pd} / \mathrm{KCC}-1$ nanocatalysts and $\mathrm{Ni} @ \mathrm{Pd} \mathrm{NPs}$ obviously showed that the Ni@Pd/KCC-1 nanocatalysts, with the easy accessibility of the active sites, exhibited higher catalytic activity than the unsupported Ni@Pd NPs. The well-dispersed nanocatalysts on mesoporous nanospheres are more stable than unsupported nanocatalysts, providing a green and environmentally benign protocol [53]. In addition, the $\mathrm{Ni} @ \mathrm{Pd} / \mathrm{KCC}-1$ nanocatalysts could be easily recovered from the reaction mixture under an external magnetic field. The recovered nanocatalysts were used for at least six runs. There was a slight trend toward deactivation of the $\mathrm{Ni} @ \mathrm{Pd} / \mathrm{KCC}-1$ nanocatalysts, probably due to the poisoning of the Pd catalyst via oxidation or catalyst inactivation. The ICP-AES analysis showed that there was little leaching of the active sites after six catalytic runs.

\subsection{Magnetic Heterodimer Catalysts}

Various hybrid nanocrystals composed of two or more different NPs have been synthesized, for the purpose of merging the properties of the individual constituents. The advantage of individual nanocrystals can be integrated in hybrid nanocrystals, exhibiting the synergistic and enhanced properties. Scheme 5 shows the three main possible formation routes of heterodimer nanocrystals. The first formation route of heterodimer nanocrystals is the phase segregation of two immiscible nanomaterials. The second synthetic approach of heterodimer nanocrystals is by coalescence of a shell nanomaterials. The third path is by selective nucleation on a formed seed. Accordingly, trimer NPs can also be made by similar paths e.g. formation of a domain with bridging two preformed NPs, fusion of two reactive domains from distinct dimers or by selective nucleation on a preformed heterodimer seed. 


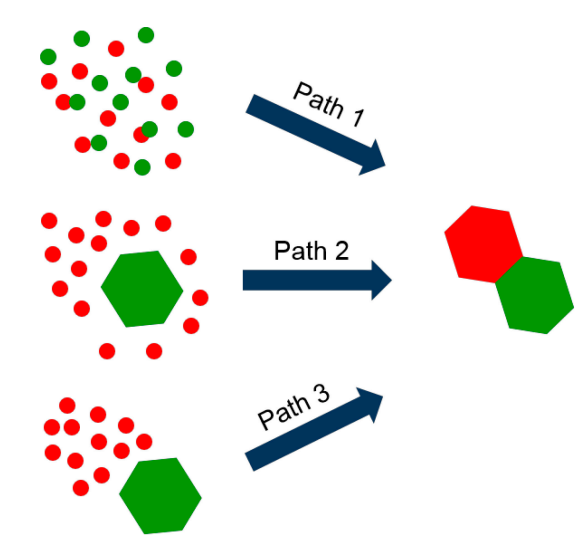

Scheme 5. Possible formation paths of heterodimers.

Heterodimeric nanocrystals with noble metals and iron oxide have been synthesized and used as reusable catalysts [54]. For example, Park and co-workers synthesized $\mathrm{Au}-\mathrm{Fe}_{3} \mathrm{O}_{4}$ heterodimer nanocrystals and applied them for the chemoselective reduction of nitro compounds [55]. The synthetic route of the most previously reported heterodimer nanocrystals involved several complex steps. To overcome this drawback, Jang et al. reported a simple and one-pot synthesis of $\mathrm{Rh}-\mathrm{Fe}_{3} \mathrm{O}_{4}$ heterodimer nanocrystals and demonstrated their applications to magnetically recyclable catalyst for the efficient and selective reduction of nitroarenes [56]. The heterodimer nanocrystals were prepared using a consecutive heating approach in a one-pot synthesis. $\mathrm{Rh}(\mathrm{acac})_{3}$ and $\mathrm{Fe}(\mathrm{acac})_{3}$ were mixed with a solution containing oleylamine and oleic acid, and the resulting mixture was slowly heated to $300{ }^{\circ} \mathrm{C}$ and aged for $30 \mathrm{~min}$. TEM images showed that the $\mathrm{Rh}-\mathrm{Fe}_{3} \mathrm{O}_{4}$ heterodimer nanocrystals consisted of $\mathrm{Rh}$ nanoparticles with an average diameter of $\sim 3 \mathrm{~nm}$ and $\mathrm{Fe}_{3} \mathrm{O}_{4}$ component of $\sim 16 \mathrm{~nm}$.

The catalytic activity of the $\mathrm{Rh}-\mathrm{Fe}_{3} \mathrm{O}_{4}$ heterodimer nanocrystals was tested for the reduction of nitrobenzene using hydrazine yielding exclusively aniline in $1 \mathrm{~h}$. To examine the scope of the reduction reaction using the $\mathrm{Rh}-\mathrm{Fe}_{3} \mathrm{O}_{4}$ heterodimer nanocrystals, the reduction of structurally diverse nitro compounds was investigated. The heterodimer nanocrystals presented excellent activity for nitro group reduction. Furthermore, an arene substrate possessing more than one nitro group could be reduced with good efficiency. The nitro group of nitroarenes with different functional groups was also selectively reduced to the amino moiety. No reduction of benzyloxy, $N$-carbobenzyloxy, ester and amide groups was observed by $\mathrm{Rh}-\mathrm{Fe}_{3} \mathrm{O}_{4}$ heterodimer nanocatalysts. In all cases examined, excellent yields of amine products were obtained as exclusive products. The $\mathrm{Rh}-\mathrm{Fe}_{3} \mathrm{O}_{4}$ heterodimer nanocrystals were easily separated by using a magnet and reused in the next reaction. It was observed that the catalysts had excellent catalytic activity at least until eight times of the catalytic reaction in the reduction of nitrobenzene under the optimized reaction conditions (Figure 5). Interestingly, the amount of $\mathrm{Rh}$ catalysts examined by ICP results before the reaction and after the eight cycles were almost the same ( $1.09 \mathrm{wt} \%$ and $1.02 \mathrm{wt} \%$, respectively), and no Rh was observed in the filtration obtained after separating the heterodimer catalyst in the first, second, fourth, sixth and eighth reactions at a detectable level. These results showed no leaching of Rh species during the reaction and recycling, as well as the consistently high catalytic efficiency of the catalyst in eight cycles. 


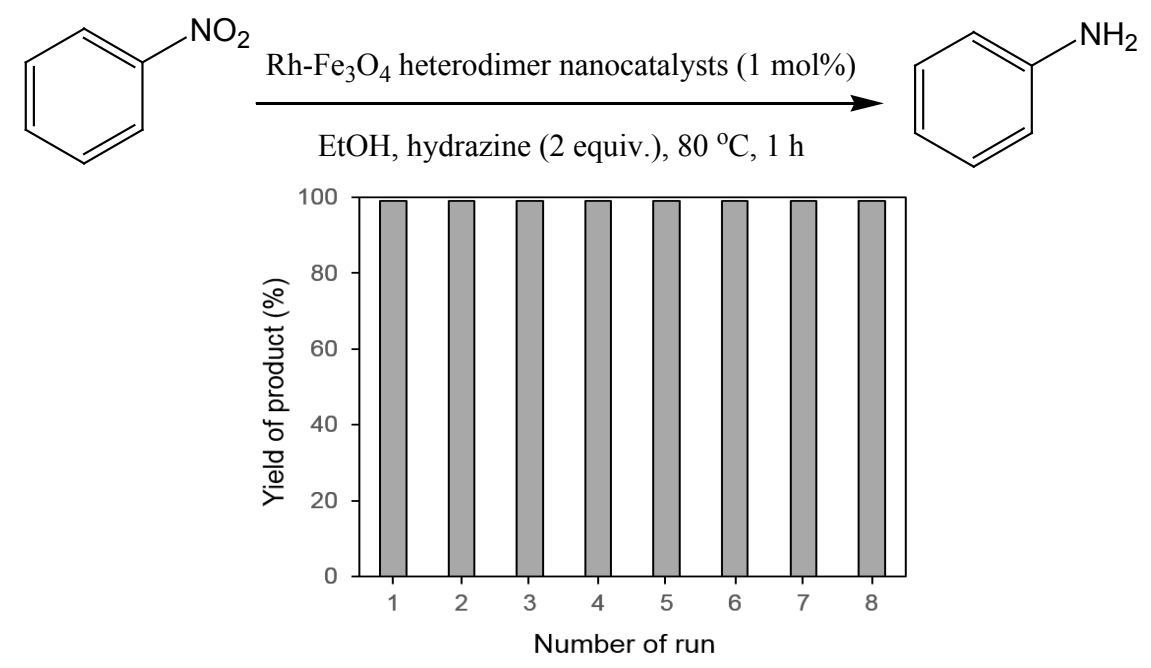

Figure 5. Recycling for the magnetic $\mathrm{Rh}-\mathrm{Fe}_{3} \mathrm{O}_{4}$ heterodimer nanocatalysts in the reduction of nitrobenzene.

\subsection{Micro-Sized Magnetic and Porous Composite Catalysts}

Nanocomposites represent an important class of nanomaterials and have attracted increased research interests due to their superior properties to individual components [57]. Although immobilizing active nanocatalysts on insoluble micro-sized solid supports maintains the catalysts activities and resolves the recycling deficiency, there are significantly lower activities or selectivities compared to homogeneous catalysts, which are commonly detected due to steric and diffusion factors. When the applied insoluble support is porous, the surface area is substantially increased, and the nanocatalysts can be evenly dispersed in the pores. Therefore, nanocatalysts do not suffer from the accessibility and other problems associated with the transport of reactants and products to the catalytic sites [58]. A combination of the aforementioned characteristics of a porous composite containing magnetic NPs and nanocatalysts can introduce a magnetic porous composite catalyst, which might present a closer characteristic to an ideal system. For example, porous carbon supports are one of the most popular matrixes as porous solid supports, because of their low cost, large surface area, availability, mechanical robustness and facile synthesis. Shokouhimehr et al. reported an economical, scalable procedure to synthesize magnetically retrievable porous carbon nanocomposite catalysts by combining magnetic NPs, nitrogen-doped porous carbon support and $\sim 3 \mathrm{~nm}$-sized catalyst NPs of Pd or Pt. The designed magnetic carbon nanocomposite catalysts provided excellent catalytic activities for the reduction of nitroarenes and Suzuki cross-coupling reactions [59]. The overall synthetic procedure to prepare the magnetically-recyclable carbon nanocomposite catalysts is illustrated in Scheme 6. The designed nanocomposite catalysts with a high surface area and permeable porous structure contain abundant and accessible small-sized catalyst NPs. These characteristics led to efficient catalytic reactions and enhanced catalytic activity.

First, a micro-sized polymer composite was synthesized via a redox reaction between pyrrole and $\mathrm{Pd}(\mathrm{OAc})_{2}$ or $\mathrm{PtCl}_{2}$ in the presence of iron oxide NPs. Pyrrole monomers could be chemically polymerized using $\mathrm{Pd}(\mathrm{II})$ or $\mathrm{Pt}(\mathrm{II})$ as oxidizing agents. Then, the magnetic carbon composite $\mathrm{Pd}$ or $\mathrm{Pt}$ catalysts were prepared via carbonization of the synthesized polymer composite. This procedure provides direct chemical reduction of $\mathrm{Pd}$ or $\mathrm{Pt}$ ions during pyrrole oxidation, so that it incorporates the 
desired nanocatalysts inside the polymer matrix and, subsequently, the carbon composite. Consequently, relatively uniform $3 \mathrm{~nm}$-sized $\mathrm{Pd}$ or Pt nanocatalysts are embedded and stabilized in an $\mathrm{N}$-doped carbon framework. TEM image (Figure 6a) of the magnetic iron oxide NPs showed that they were relatively uniform and spherical, with an average diameter of $\sim 18 \mathrm{~nm}$. The TEM image of the polymer (Figure $6 \mathrm{~b}$ ) and carbon (Figure 6c) composite catalysts revealed that Pd NPs and Pt NPs were crystallized with an average distribution size of $3 \mathrm{~nm}$. The HRTEM image (Figure 6d) clearly demonstrated the porous carbon matrix, which is an important parameter for the accessibility of reactants to the nanocatalysts. The designed magnetically recyclable carbon composite could be readily used as general framework supports for loading other noble metal NPs, such as palladium or platinum, which can be utilized for different catalytic reactions.

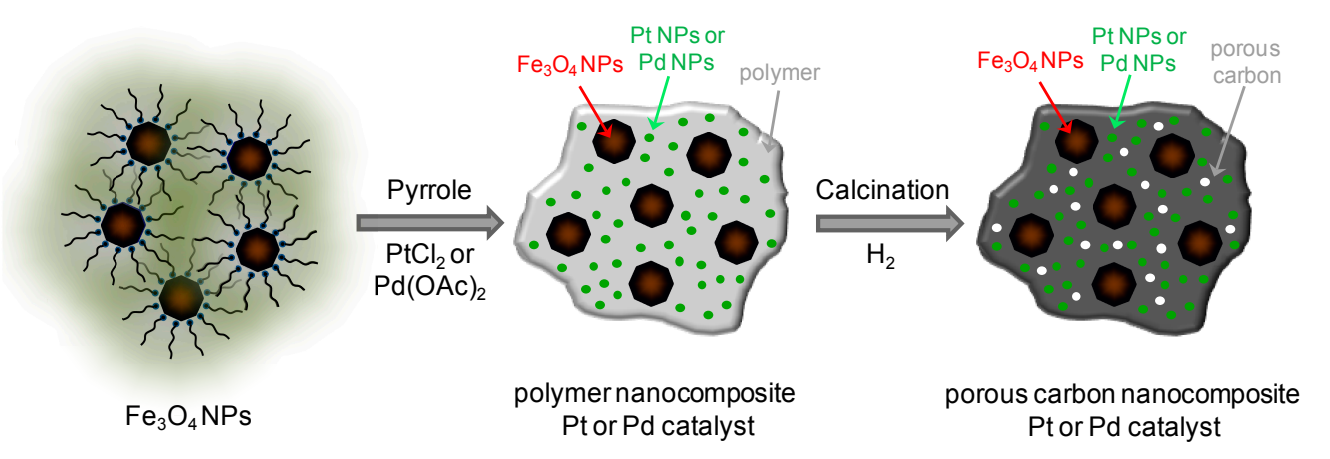

Scheme 6. Synthetic procedure of the magnetically-recyclable porous carbon nanocomposite catalysts. Reprinted with permission from [59].

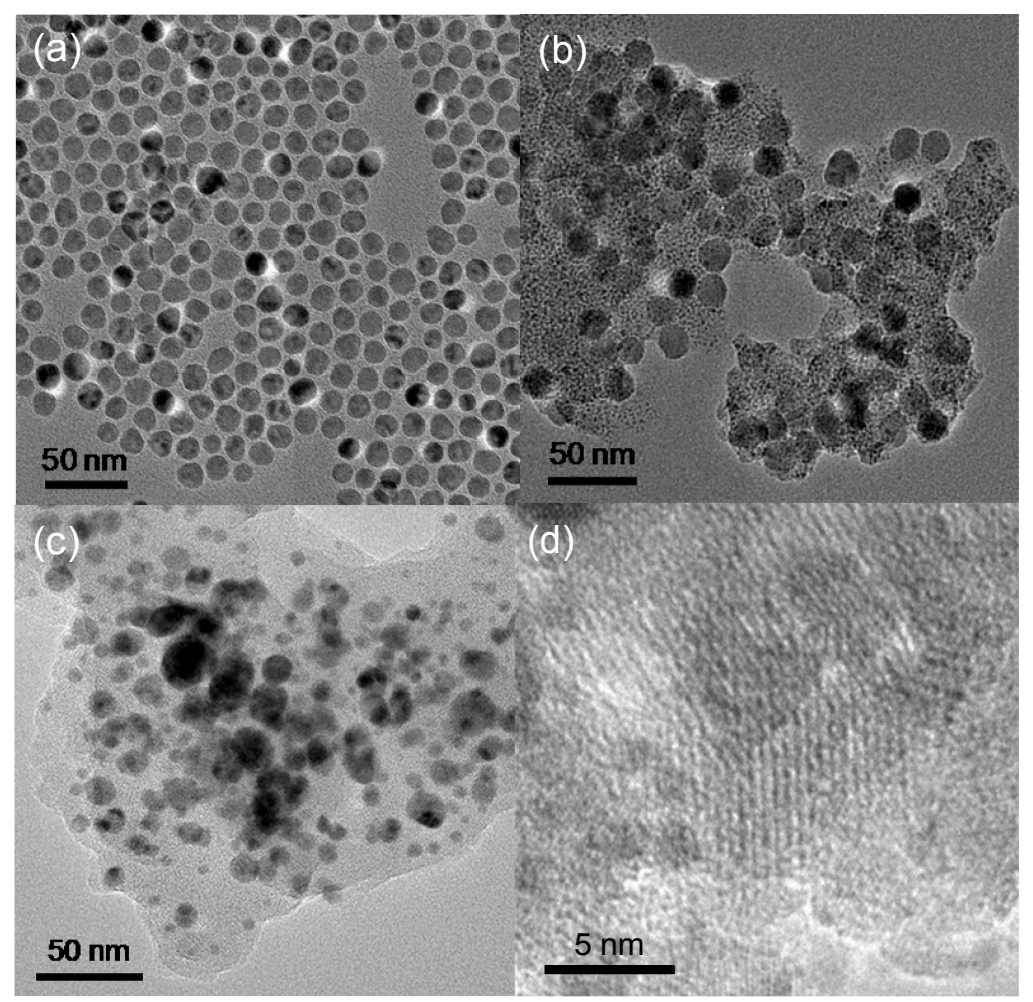

Figure 6. (a) TEM image of $\mathrm{Fe}_{3} \mathrm{O}_{4} \mathrm{NPs}$; (b) TEM image of polymer composite catalyst; (c) TEM image of carbon composite catalyst; (d) HRTEM image of the porous carbon matrix. Reprinted with permission from [59]. 
The catalytic activity of the Pt composite catalysts was investigated for the reduction of structurally-diverse nitro compounds. When the catalytic performance of the Pt composite catalyst was tested for the reduction of nitrobenzene using hydrazine, the reaction was completed in $3 \mathrm{~h}$, yielding quantitative aniline (Table 2, Entry 1). In an attempt to extend the application of this catalyst, the reduction of structurally diverse nitro compounds was studied. Table 2 confirms that the Pt composite catalyst exhibited excellent activities for the reduction of nitrobenzene derivatives. The nitro group of nitroarenes with different functional groups using the magnetically-recyclable composite catalyst was selectively reduced to the amino moiety (Table 2, Entries 2-4). The halogen functional groups remained intact under the reaction conditions. In all cases examined in Table 2, the excellent yields of amine products were obtained as exclusive products.

Table 2. Heterogeneous reduction of substituted nitrobenzene with hydrazine. Reprinted with permission from [59].

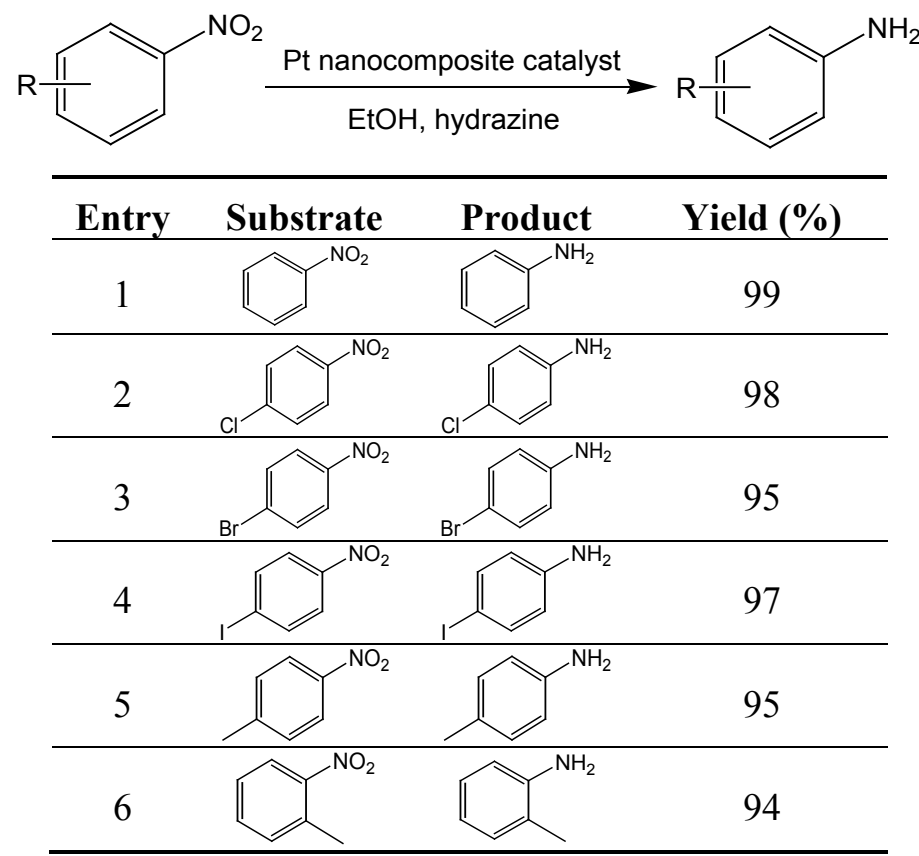

Reaction conditions: substituted nitrobenzene $(0.5 \mathrm{mmol})$, hydrazine (2 equiv.), Pt nanocomposite catalyst (1 mol\%), EtOH, $80^{\circ} \mathrm{C}, 3 \mathrm{~h}$.

To verify the reusability and durability issues, the magnetic separation and recycling of the magnetically recyclable $\mathrm{Pt}$ composite catalyst was investigated under the optimized reaction conditions (0.5 mmol nitrobenzene, 2 equiv. hydrazine, $1 \mathrm{~mol} \% \mathrm{Pt}$ nanocomposite catalyst, $\mathrm{EtOH}, 80{ }^{\circ} \mathrm{C}$ ), which was successfully recycled and reused for five consecutive cycles of the reduction of nitrobenzene with no significant loss of activity (Table 3). Magnetic recovery could eliminate the tedious work-up and catalyst filtration procedures after completion of the reactions. Upon the completion of the reaction, the catalyst was easily separated using a magnet, washed with EtOH and reused in the next reaction.

The designed composites with a well-defined porous carbon matrix and high surface area accommodated abundant $3 \mathrm{~nm}$-sized NPs of Pd or Pt, providing excellent catalytic activities in a short reaction time. Furthermore, the composite catalyst could be readily synthesized in a large scale by a simple and inexpensive process and used as a general platform to load various catalytic NPs for different catalytic reactions. 
Table 3. Magnetic separation and recycling of the $\mathrm{Pt}$ nanocomposite catalyst in the heterogeneous reduction of nitrobenzene with hydrazine under the optimized reaction conditions. Reprinted with permission from [59].

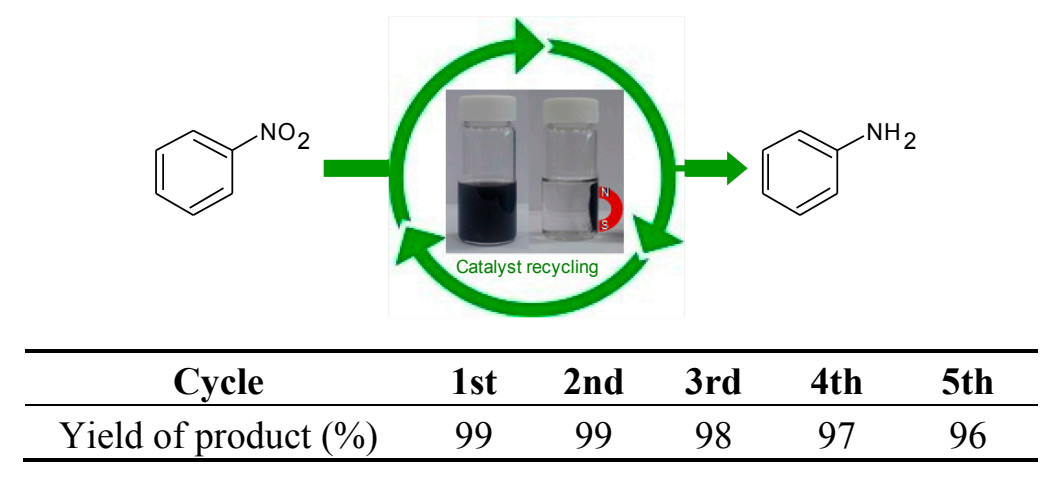

\subsection{Magnetic Hollow Nanostructured Catalysts}

Hollow nanostructures are considered as a very important category of nanomaterials and have been widely investigated and applied in many research fields, such as adsorption and energy storage. Hollow nanocapsules possess high surface areas, low density and self-supporting capacity compared to their solid counterparts. They can be incorporated with catalytically-active NPs, provide dynamic catalytic activity and consequently hold promising applications as nanoreactors for catalytic reactions. Therefore, they have emerged as rapidly growing research themes and have been widely applied in the field of catalysis [60]. However, the typical synthetic protocols for hollow nanostructures, such as the well-known hard-template methods and surface-protected etching, have limited success in large amount production, structure morphology and engineering and aggregation-free nanostructures. Despite being widely used in the catalytic transformations, hollow nanostructure catalysts are generally unstable, and structural collapse occurs after a single use in catalytic reactions, causing a rapid catalytic activity decay. Similar to the non-magnetic systems, these nanostructures also generally require a centrifugation step or a tedious workup of the final reaction mixture to recover the catalyst [61]. To overcome these shortcomings, Wang et al. stabilized Pd NPs on amine-functionalized hollow magnetite NPs and utilized them for hydrogenation reactions [62]. Recently, in a practical approach, Shokouhimehr and co-workers introduced an economical procedure to prepare stable, scalable and recyclable hollow nanostructures as catalyst supports to avoid the existing problems of these nanostructures. They developed a method by combining magnetic and self-assembled hollow structural properties for the synthesis of highly sustainable, magnetically-recyclable hollow nanostructure catalysts. The designed catalysts give excellent catalytic activities for the reduction of nitroarenes [63]. The overall synthetic procedure is represented in Scheme 7.

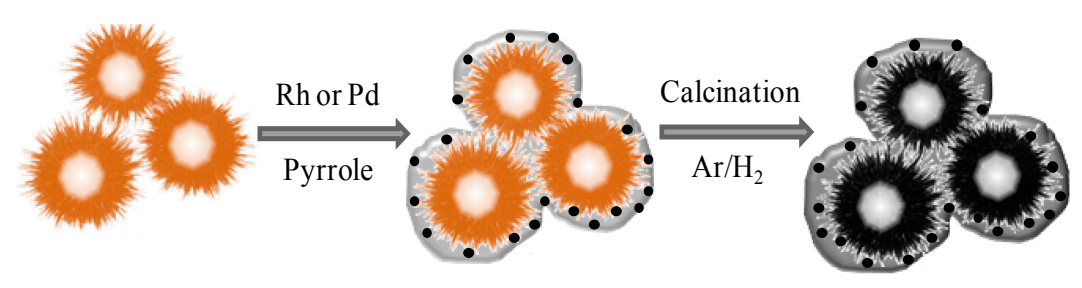

Scheme 7. Synthetic procedure leading to the magnetically-recyclable hollow nanostructured catalyst. Reprinted with permission from [63]. 
First, the hollow iron hydroxide nanostructure was prepared by a template-free and simple one-pot reaction. A carbon precursor was introduced in the ongoing reaction and chemically polymerized by a palladium or rhodium precursor as an oxidant. Then, the magnetically-recyclable hollow nanostructured catalysts were prepared via a calcination step. The TEM image of the Rh nanostructured catalyst showed that Rh NPs were uniform with an average size of $\sim 2 \mathrm{~nm}$ (Figure $7 \mathrm{a}$ ). The presence of $\mathrm{Rh}$ NPs on the nanostructure was verified using XPS analysis (Figure 7b). The EDX of this indicated that the Rh species were successfully immobilized on the nanocomposite (Figure 7c).

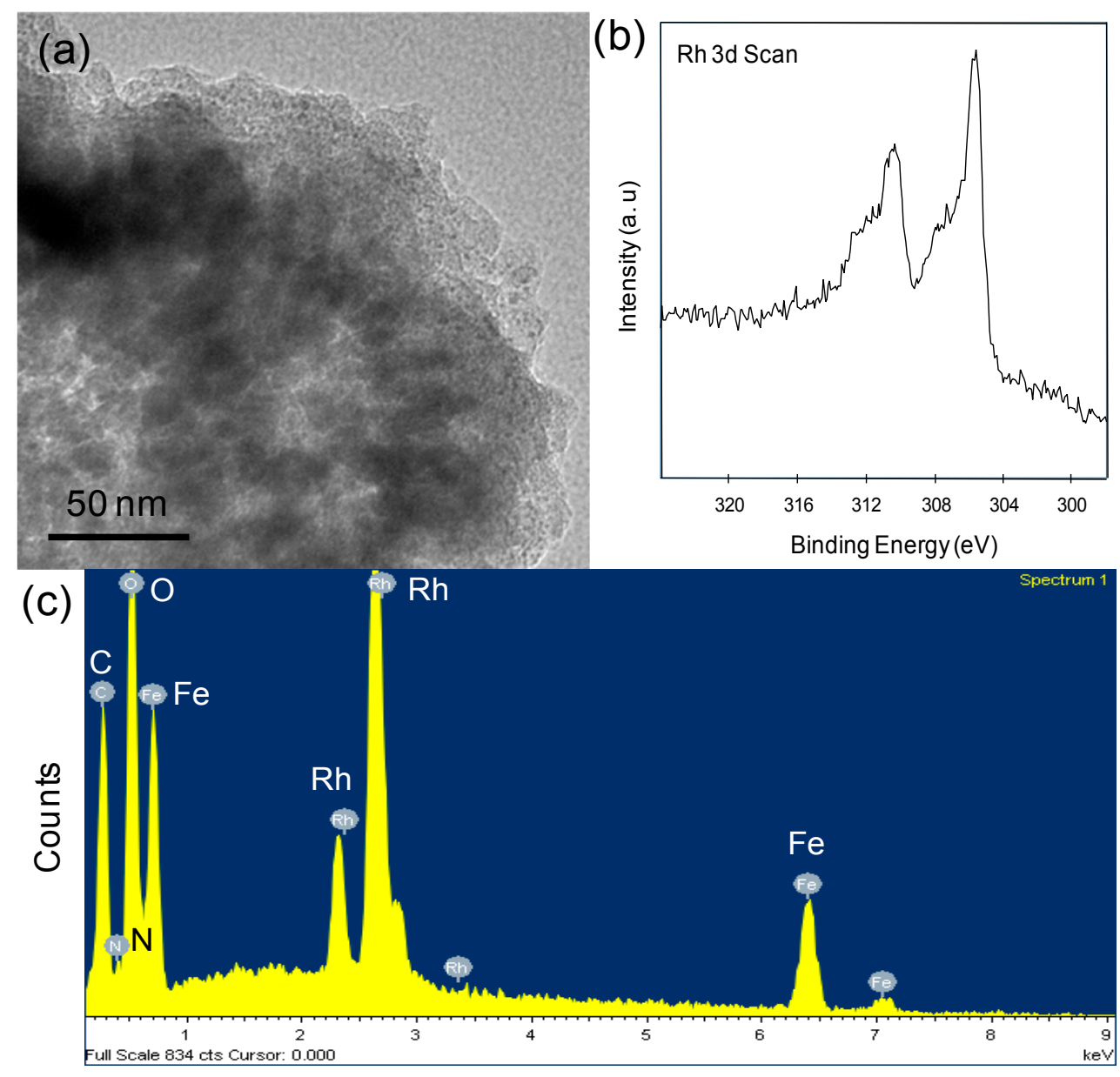

Figure 7. (a) TEM images of magnetically-recyclable hollow nanostructured Rh catalyst; (b) XPS analysis of Rh 3d scan; (c) EDX spectrum of magnetically-recyclable hollow nanostructured Rh catalyst. Reprinted with permission from [63].

The carbon coating kept the hollow nanostructure catalysts from structural collapse during the calcination step and catalytic reactions. In addition, the porous carbon layer offered permeable paths to organic reagents and improved the diffusion of the substrates/products. Nitrogen-containing carbon could also enhance the interaction between nanocatalysts and the carbon surface. The well-defined carbon matrix could serve as a shield and stabilize catalyst NPs to maintain their activities in harsh reaction conditions, such as temperature alteration, stirring and shaking.

The catalytic activity of the designed $\mathrm{Rh}$ catalyst was examined in the reduction of structurally-diverse nitro compounds. Table 4 confirms that the Rh nanocrystals had excellent activity in the nitro reduction. Although the selective reduction of a nitro group in organic compounds containing 
other reducible functional groups is a challenging reaction, the nitro group of nitroarenes with different functional groups using this catalyst was selectively reduced to the amino moiety (Table 4, Entries 2-5). The halogen functional groups remained intact under the reaction conditions. The designed hollow nanostructure produced a high surface area and accommodated a large amount of small-sized nanocatalysts, leading to the high catalytic activity. The magnetic separation of the catalyst is much more convenient for the catalyst recycling than the filtration and centrifugation methods. These attractive features exhibited by the designed magnetically-recyclable hollow nanostructured catalysts enabled having high catalytic activity for the reduction of nitroaromatics in mild reaction conditions and a short reaction time.

Table 4. Heterogeneous reduction of nitrobenzene derivatives with hydrazine. Reprinted with permission from [63].

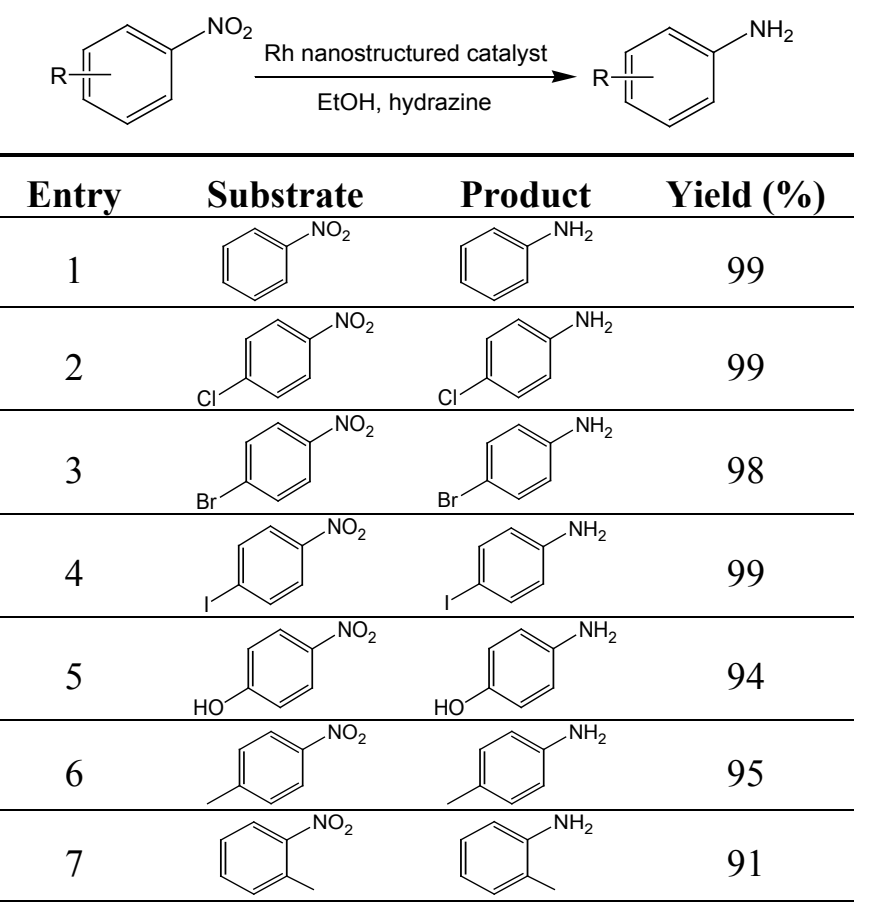

Reaction conditions: substituted nitrobenzene $(0.5 \mathrm{mmol})$, hydrazine (2 equiv.), Rh catalyst ( $1 \mathrm{~mol} \%)$, $\mathrm{EtOH}, 80^{\circ} \mathrm{C}, 2.5 \mathrm{~h}$.

The magnetic properties of the nanostructure could be readily utilized for the easy recovery and recycling of the catalysts. The catalysts could be reused for five consecutive cycles in the reduction of nitrobenzene with no major loss of activity. Upon the completion of the reaction, the catalyst was easily separated using a magnet and reused in the next reaction. The magnetic separation and recycling of the magnetically-recyclable hollow nanostructured $\mathrm{Rh}$ catalyst (1 mol\%) was studied in the reduction of nitrobenzene $(0.5 \mathrm{mmol}$ ) using hydrazine ( 2 equiv.) under the optimized reaction conditions, which was successfully reused for five consecutive cycles with no major loss of activity (Table 5). Upon the completion of the reaction, the catalyst was easily separated using a magnet and reused in the next reaction. The magnetically-recyclable hollow catalysts could be also synthesized in a large scale using a simple process. When larger amounts of starting materials were used, $\sim 2.3 \mathrm{~g}$ of the nanocomposite catalyst could be obtained by one-batch synthesis. 
Table 5. Magnetic separation and recycling of the magnetically-recyclable hollow nanostructured $\mathrm{Rh}$ catalyst in the heterogeneous reduction of nitrobenzene with hydrazine under the optimized reaction conditions. Reprinted with permission from [63].

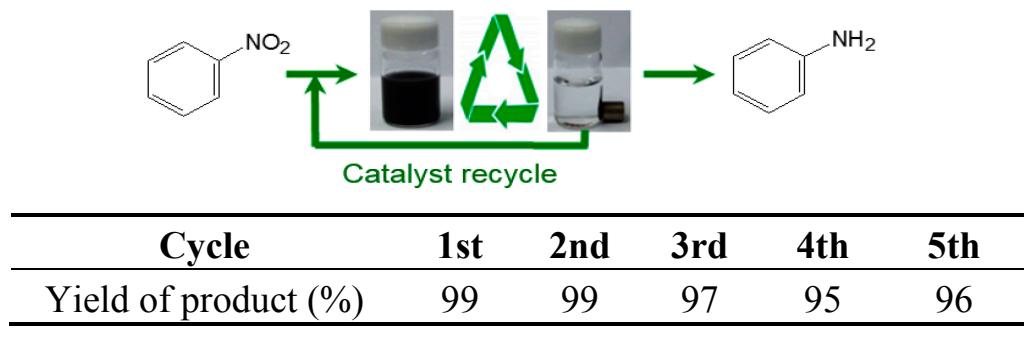

\subsection{Magnetic Core-Shell and Yolk-Shell Nanostructured Catalysts}

Modern nanotechnology and techniques allow significant improvements in the synthesis and structural design of enhanced catalysts with precise shape, size and surface properties. In particular, the remaining challenges, e.g., improving the structural stability, efficiency and durability of the engineered catalysts, can be rectified [64]. The syntheses, characterizations and practical applications of effective core-shell and yolk-shell nanostructured catalysts have been introduced as a unique strategy to resolve the aforementioned problems. Core-shell nanostructure catalysts are generally encapsulated and protected by an outer shell that isolates the catalytic NPs and prevents their migration and coalescence during the catalytic reactions [65]. Yolk-shell nanostructured catalysts consist of a core encapsulated in a hollow capsule with a permeable shell, and catalytic NPs are designed as an alternative tactic to meet an excellent catalyst [66]. Gawande et al. prepared magnetically-separable $\mathrm{Ag} @ \mathrm{Ni}$ core-shell nanocatalysts by a simple one-pot synthesis route and verified their catalytic activity in hydrogenation reactions [67]. The recyclable nanocatalysts presented excellent activity for the reduction of aromatic nitro compounds under mild conditions using isopropyl alcohol as a hydrogen donor. Choi and co-workers stabilized Pd nanocatalysts on magnetic core-shell nanostructures to prevent them from sintering and coagulation [68]. The magnetic core could be readily utilized for the easy recovery and recycling of the nanocatalysts. To prepare highly stable and practically effective core-shell catalysts, Shokouhimehr et al. designed and synthesized a magnetically-recyclable core-shell with the robustly fixed Pd nanocatalysts on a polymer layer for the efficient oxidation of alcohols, Suzuki cross-coupling and reduction of nitroaromatics [69]. The Pd NPs half partitioned in the polymer matrix could provide not only high catalytic activity, but also stabilization of the nanocatalysts under harsh reaction conditions. The designed nanocatalysts presented excellent catalytic activities for the above-mentioned catalytic transformations. The overall synthetic procedure is shown in Scheme 8 . The magnetic core was utilized for the magnetic recycling of the Pd nanocatalysts.

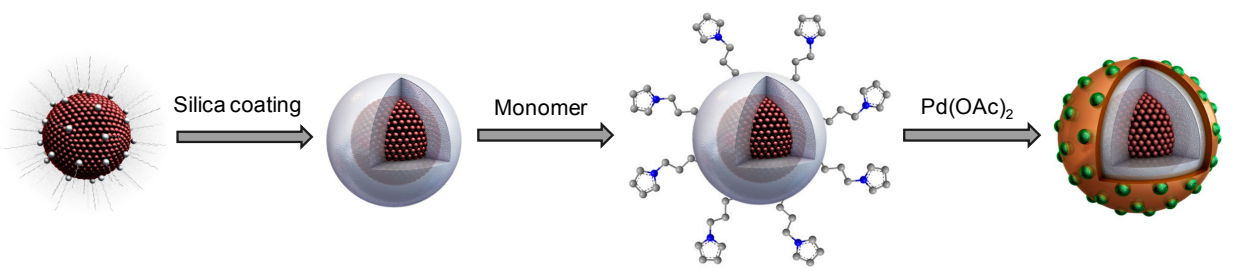

Scheme 8. Schematic preparation of the magnetically-recyclable core-shell nanocatalysts. Reprinted with permission from [69]. 
First, iron oxide NPs were synthesized using a previously reported method [70]. As-synthesized $\mathrm{Fe}_{3} \mathrm{O}_{4}$ NPs were dispersed in cyclohexane containing igepa ${ }^{\circledR} \mathrm{CO}-520$, and an aqueous ammonia solution was directly added. Tetraethoxysilane was then rapidly added, and the solution was vigorously stirred at room temperature to obtain silica-coated iron oxide NPs. After ageing, the pyrrole functionalized silica shell was formed by the addition of $N$-(3-trimethoxysilylpropyl)-pyrrole for $3 \mathrm{~h}$. The product was isolated by centrifugation, washed with ethanol and dispersed in ethanol. While vigorously stirring, palladium(II) acetate dissolved in chloroform-ethanol was added dropwise to the functionalized core-shell NPs, and the mixture was stirred for $3 \mathrm{~h}$ at room temperature to produce the magnetically-recyclable core-shell nanocatalysts. The color of the mixture changed from light brown to deep black, indicating the reduction of $\mathrm{Pd}(\mathrm{OAc})_{2}$ by a pyrrole moiety. The product was washed several times with chloroform-ethanol and dried in vacuo. The palladium loading of the product was measured by ICP-AES.

The designed core-shell nanocatalysts were intensively characterized. The TEM images of the magnetic iron oxide NPs showed that they were uniform spheres with a size of $20 \mathrm{~nm}$ (Figure 8a). TEM and HRTEM images of silica-coated $\mathrm{Fe}_{3} \mathrm{O}_{4}$ NPs indicated uniform core-shell nanostructures with a thickness of $\sim 15 \mathrm{~nm}$ (Figure $8 \mathrm{~b}$ ). TEM and HRTEM images also revealed the relatively uniform Pd NPs of $\sim 3 \mathrm{~nm}$ in diameter incorporated discretely in a thin polymer layer deposited on magnetic silica core-shell nanospheres (Figure 8c,d). Field emission scanning electron microscopy (FESEM) images demonstrated that the magnetically-recyclable Pd nanocatalysts exhibited a rough surface due to the accommodation of the Pd NPs in the polymer layer, whereas the dense silica-coated magnetic NPs appeared to have a smooth surface (Figure 8e,f).

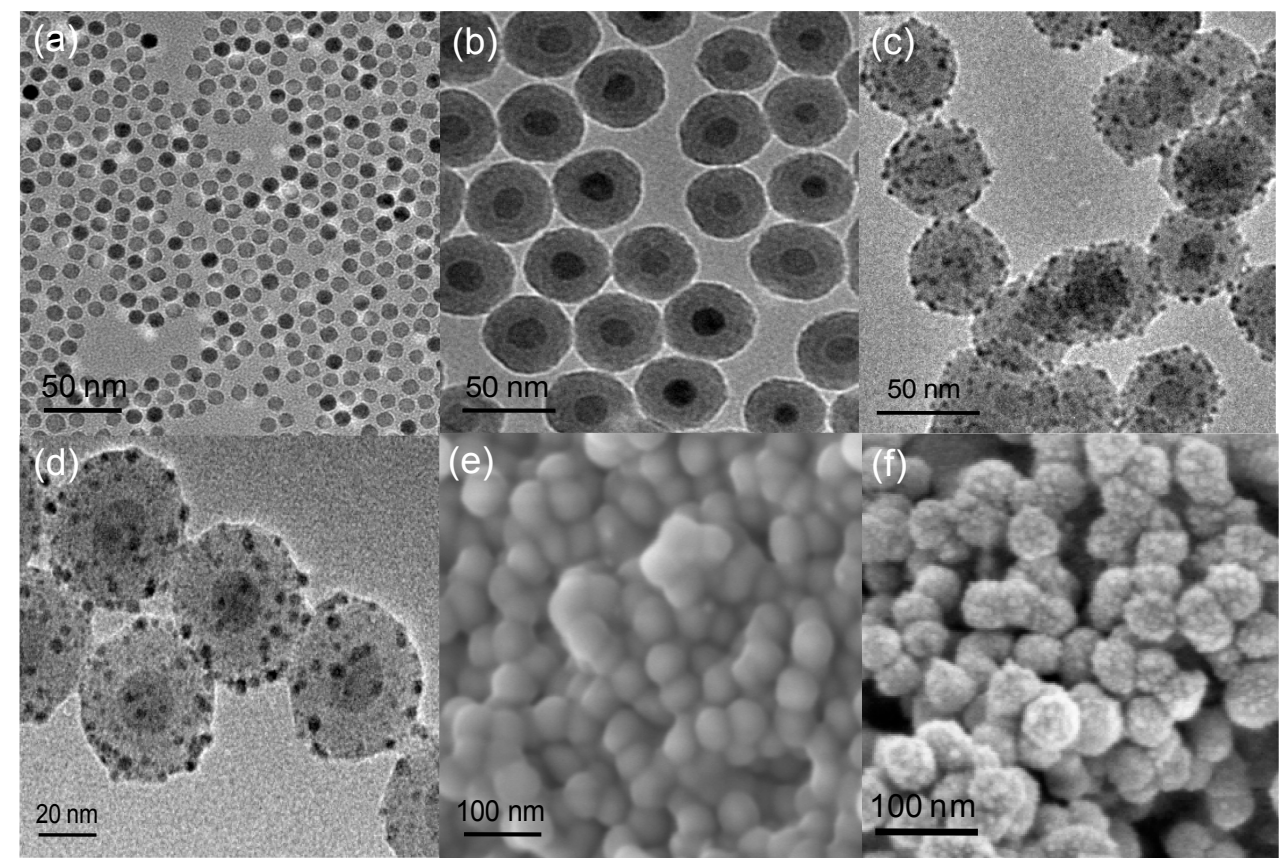

Figure 8. (a) TEM image of $\mathrm{Fe}_{3} \mathrm{O}_{4} \mathrm{NPs}$; (b) TEM image of silica-coated $\mathrm{Fe}_{3} \mathrm{O}_{4} \mathrm{NPs}$; (c) TEM image of the magnetically-recyclable Pd nanocatalysts; (d) HRTEM image of the magnetically-recyclable Pd nanocatalysts; (e) FESEM image of silica-coated $\mathrm{Fe}_{3} \mathrm{O}_{4} \mathrm{NPs}$; (f) FESEM image of the magnetically-recyclable Pd nanocatalysts. Reprinted with permission from [69]. 
The designed core-shell nanostructure could improve the interaction and reactivity of the Pd nanocatalysts. The impermeable silica layer isolated the core magnetic NPs and prevented the possibility of side reactions during the processes. In addition, any synergistic effect of the catalytic NPs and the support can be promoted when such interfaces are important in catalytic performances. The magnetic separation provides a convenient method for removing and recycling the active $\mathrm{Pd}$ nanocatalysts from the reaction mixture. The designed nanocatalysts showed excellent activity in the heterogeneous reduction of nitro compounds (Table 6). The halogen functional groups remained intact under the reaction conditions. The nitro group of nitroarenes with different functional groups using the magnetically-recyclable nanocatalysts was selectively reduced to the amine moiety. Although the reusability of the magnetic core-shell nanocatalysts was verified for the five consecutive cycles of the oxidation of cycloheptanol, the authors did not report the results of recycling for the Suzuki cross-coupling reaction and reduction of nitroaromatics.

Table 6. Heterogeneous reduction of substituted nitrobenzene. Reprinted with permission from [69].

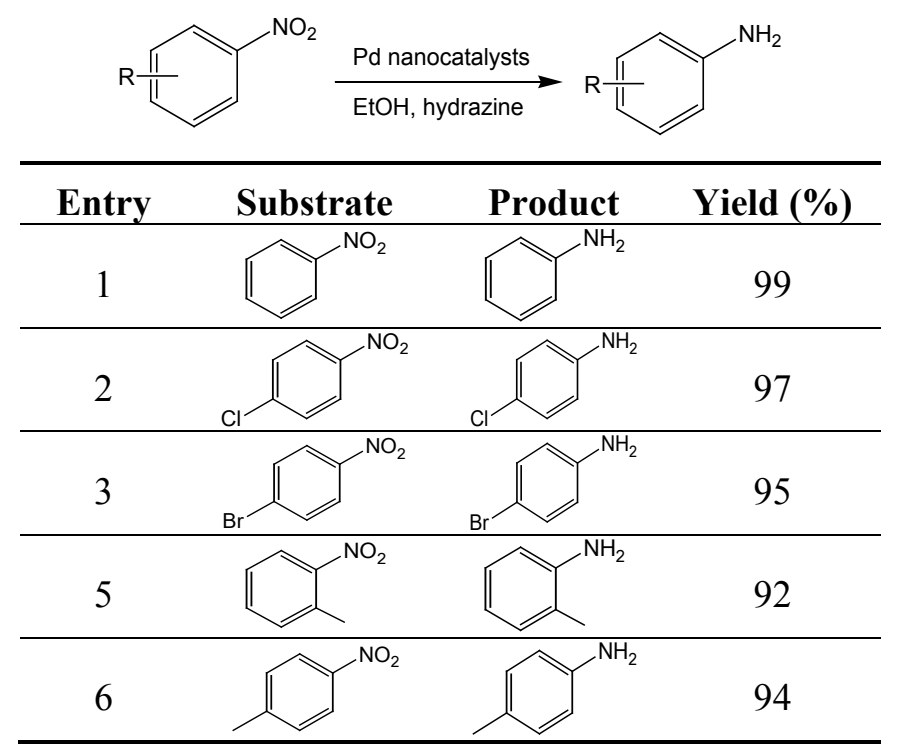

Reaction conditions: substituted nitrobenzene ( $1 \mathrm{mmol}$ ), hydrazine (2 equiv.), Pd nanocatalyst ( $1 \mathrm{~mol} \%)$, EtOH, $80^{\circ} \mathrm{C}, 4 \mathrm{~h}$.

Yolk-shell nanostructures have generated recent research interest because of their potential applications in heterogeneous catalysis. A major problem of the yolk-shell catalysts that needs to be resolved is the requirement of tedious filtration or centrifugation during the recovery and reuse, which greatly limits their practical applicability. Yolk-shell nanostructured catalysts with magnetic NP interior spaces represent a promising framework for the development of catalytic nanoreactor systems with high activity, selectivity and recyclability, which can tackle the issues of conventional nanocatalysts [71]. Yao et al. introduced a facile method to fabricate yolk-shell composites with an iron oxide core and mesoporous $\mathrm{SiO}_{2}$ shell with Pd NPs anchoring on the inner surface and studied their catalytic activity in the reduction of 4-nitrophenol [72]. The inner $\mathrm{Fe}_{3} \mathrm{O}_{4}$ NP core of the yolk-shell endowed the yolk-shell nanoreactors with superparamagnetism and, thereby, simplified the introduction procedure of a magnetic component. The mesoporous shell could not only allow the fast diffusion of reactants and products, but also effectively protects the NPs from escaping outside and being corroded by the solution. 
To synthesize the magnetic yolk-shell microsphere catalysts, first, monodispersed $\mathrm{Fe}_{3} \mathrm{O}_{4} \mathrm{NPs}_{\text {s }}$ were synthesized according to the previous work [73]. As-prepared $\mathrm{Fe}_{3} \mathrm{O}_{4}$ nanospheres and $2.5 \mathrm{~g}$ glucose were dispersed in water, transferred into an autoclave and sealed to heat at $200{ }^{\circ} \mathrm{C}$ for $8 \mathrm{~h}$. After washing the obtained products, they were added into a $\mathrm{PdCl}_{2} / \mathrm{EtOH}$ solution under stirring, and then, hydrazine was added to the mixture. Then, the obtained nanocomposites were dispersed into the mixture of the $\mathrm{H}_{2} \mathrm{O} / \mathrm{EtOH}$ containing hexadecyl trimethyl ammonium bromide and ammonia. After mechanically stirring for $0.5 \mathrm{~h}, 150 \mathrm{~mL}$ of tetraethyl orthosilicate were added into the system and kept for $6 \mathrm{~h}$. The products were collected by a magnet and dried in an oven. Finally, the nanocomposites were heated at $600{ }^{\circ} \mathrm{C}$ for $3.0 \mathrm{~h}$, and the yolk-shell nanocomposite catalysts were prepared (Scheme 9).

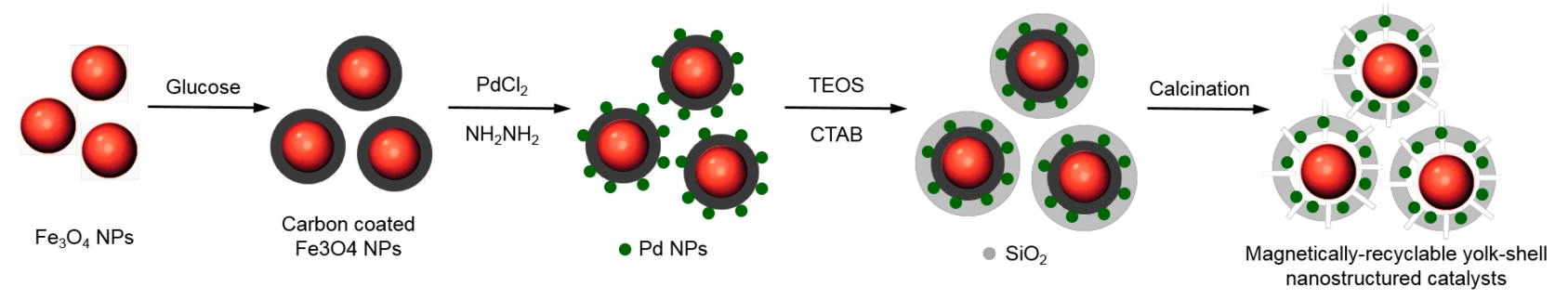

Scheme 9. Synthetic procedure for the magnetically-recyclable yolk-shell nanostructured catalysts.

The TEM images confirm that the magnetic nanostructured catalysts possess a well-defined yolk-shell structure. The $\mathrm{N}_{2}$ adsorption-desorption isotherms of the yolk-shell catalyst exhibited representative Type IV curves, and their BET surface area is about $270 \mathrm{~m}^{2} \cdot \mathrm{g}^{-1}$. From the pore size distribution, it could be seen that the pores mainly range from 2-4 nm with a sharp peak appearing at around $2.5 \mathrm{~nm}$.

The reduction of 4-nitrophenol was explored as a standard reaction for determining the catalytic activity of the designed yolk-shell catalysts. The preliminary catalytic testing was carried out by reducing 4-nitrophenol in water with $\mathrm{NaBH}_{4}$ as the reducing agent. The reaction rate of 4-nitrophenol reduction in the presence of $\mathrm{NaBH}_{4}$ solution catalyzed by $2 \mathrm{mg}$ of yolk-shell nanocatalysts was too quick to be monitored. Therefore, the amount of catalysts was reduced to $0.1 \mathrm{mg}$. The synthesized yolk-shell nanocatalysts could be easily separated from the solution by using a magnet when the reduction reaction completely finished due to the magnetic properties and good stability. After washing with water, they were reused in the next reaction run. The procedure was repeated 10 times, and no significant decrease in catalytic activity was found during the recycling test. Besides studying the catalytic property of yolk-shell nanocatalysts by the cycling test, the authors also investigated their integrity after 10 successive cycles by using SEM and TEM analyses. Compared with the initial materials, there was no obvious difference in both the yolk-shell structure and high dispersity of Pd NPs, which further confirmed the good stability of the yolk-shell nanocatalysts.

Incorporating magnetic properties with nanostructured catalysts is a practical method to develop sustainable catalytic systems. It not only minimizes the possibility of catalyst aggregation during recovery, but also improves the durability of the catalysts. Although magnetic recycling, compared to other procedures, obviates the requirement of filtration and provides a facile technique for reusing catalysts, it suffers from several drawbacks, such as sophisticated preparation methods and demanding large-scale synthesis procedures. Developing a simple method to produce a large amount of magnetic 
nanocomposite catalysts is very challenging, because each constituent material has different physicochemical properties and requires different reaction conditions. In addition, the complicated modification steps may decrease the magnetization and stability of the magnetic NPs, which reduce the catalyst recovery and, hence, the catalytic efficacy. The catalytic activity decay in the magnetic heterogeneous catalytic systems is also the result of the catalyst loss during the magnetic separation or leaching of catalytic species. The magnetic nanostructured catalysts also retain the issues of other heterogeneous systems, e.g., catalyst accessibility hindrance, diffusion of reactants, chemo- and regio-selectivity, low yield of products, harsh reaction conditions and long reaction times. Continuous advancements in synthesis, characterizations and applications can enhance the performances of magnetic nanostructured catalysts and resolve their problems. These catalysts can be very important for their future industrial applications in numerous important catalytic processes.

\section{Conclusions}

Heterogeneous catalysis with magnetically-recyclable nanostructured catalysts is a rapidly growing field for the development of sustainable and green processes. The application of the magnetically-reusable nanostructured catalysts is attracting great attention in the reduction of nitroarenes, because this process is a very important chemical transformation for the production of a large variety of important intermediates in the laboratory and industry. It is also an important issue to design the strategies for the synthesis of reusable magnetic nanostructured catalysts in order to prevent aggregation and achieve highly durable catalysts. This review summarizes the designed magnetic nanostructured catalysts with the enhanced and sustainable catalytic performances for the heterogeneous reduction of nitoaromatics. The preparation methods, recyclability and stability of various recyclable magnetic nanostructured catalysts are highlighted.

\section{Conflicts of Interest}

The authors declare no conflict of interest.

\section{References}

1. Tafesh, A.M.; Weiguny, J. A review of the selective catalytic reduction of aromatic nitro compounds into aromatic amines, isocyanates, carbamates, and ureas using CO. Chem. Rev. 1996, 96, 2035-2052.

2. Cenini, S.; Ragaini, F. Catalytic Reductive Carbonylation of Organic Nitro Compounds; Kluwer Academic Publisher: Dordrecht, The Netherlands, 1997.

3. Blaser, H.U.; Malan, C.; Pugin, B.; Spindler, F.; Steiner, H.; Studer, M. Selective hydrogenation for fine chemicals: Recent trends and new developments. Adv. Synth. Catal. 2003, 345, 103-151.

4. Wu, H.; Zhuo, L.; He, Q.; Liao, X.; Shi, B. Heterogeneous hydrogenation of nitrobenzenes over recyclable $\operatorname{Pd}(0)$ nanoparticle catalysts stabilized by polyphenol-grafted collagen fibers. Appl. Catal. A 2009, 366, 44-56.

5. Gelder, E.A.; Jackson, S.D.; Lok, C.M. The hydrogenation of nitrobenzene to aniline: A new mechanism. Chem. Commun. 2005, 522-524. doi:10.1039/b411603h. 
6. Verho, O.; Gustaffson, K.P.J.; Nagendiran, A.; Tai, C.W.; Backvall, J.E. Mild and selective hydrogenation of nitro compounds using palladium nanoparticles supported on amino-functionalized mesocellular foam. Chem CatChem 2014, 6, 3153-3159.

7. Westerhaus, F.A.; Jagadeesh, R.V.; Wienhofer, G.; Pohl, M.M.; Radnik, J.; Surkus, A.E.; Rabeah, J.; Junge, K.; Junge, H.; Nielsen, M.; et al. Heterogenized cobalt oxide catalysts for nitroarene reduction by pyrolysis of molecularly defined complexes. Nat. Chem. 2013, 5, 527-543.

8. Rahaim, R.J.; Maleczka, R.E. Pd-catalyzed silicon hydride reductions of aromatic and aliphatic nitro groups. Org. Lett. 2005, 7, 5087-5090.

9. Shil, A.K.; Das, P. Solid supported platinum $(0)$ nanoparticles catalyzed chemo-selective reduction of nitroarenes to $N$-arylhydroxylamines. Green Chem. 2013, 15, 3421-3428.

10. Schabel, T.; Belger, C.; Plietker, B. A mild chemoselective Ru-catalyzed reduction of alkynes, ketones, and nitro compounds. Org. Lett. 2013, 15, 2858-2861.

11. Coperet, C.; Chabanas, M.; Saint-Arroman, R.P.; Basset, J.M. Homogeneous and heterogeneous catalysis: Bridging the gap through surface organometallic chemistry. Angew. Chem. Int. Ed. 2003, 42, 156-181.

12. Shokouhimehr, M.; Kim, J.H.; Lee, Y.S. Heterogeneous Heck reaction catalyzed by recyclable polymer-supported $N$-heterocyclic carbine-palladium complex. Synlett 2006, 4, 618-620.

13. Janssen, M.; Muller, C.; Voget, D. Recent advances in the recycling of homogeneous catalysts using membrane separation. Green Chem. 2011, 13, 2247-2257.

14. Kim, J.H.; Kim, J.W.; Shokouhimehr, M.; Lee, Y.S. Polymer-supported N-heterocyclic carbene-palladium complex for heterogeneous Suzuki cross-coupling reaction. J. Org. Chem. 2005, $70,6714-6720$.

15. Luo, P.; Xu, K.; Huang, L.; Wang, J.; Xing, W.; Huang, J. Highly efficient and selective reduction of nitroarenes with hydrazine over supported rhodium nanoparticles. Catal. Sci. Technol. 2012, 2 , 301-304.

16. Polshettiwar, V.; Len, C.; Fihri, A. Silica-supported palladium: Sustainable catalysts for cross-coupling reactions. Coord. Chem. Rev. 2009, 253, 2599-2626.

17. Toebes, L.M.; Dillen, J.A.V.; Jong, K.P.D. Synthesis of supported palladium catalysts. J. Mol. Catal. A 2001, 173, 75-98.

18. Campbell, T.C.; Seller, J.R.V. Anchored metal nanoparticles: Effects of support and size on their energy, sintering resistance and reactivity. Faraday Discuss. 2013, 162, 9-30.

19. Rafiaei, M.S.; Kim, A.; Shokouhimehr, M. Gadolinium triflate immobilized on magnetic nanocomposites as recyclable Lewis acid catalyst for acetylation of phenols. Nanosci. Nanotechnol. Lett. 2014, 6, 309-313.

20. Piao, Y.; Jang, Y.; Shokouhimehr, M.; Lee, I.S.; Hyeon, T. Facile aqueous-phase synthesis of uniform palladium nanoparticles of various shapes and sizes. Small 2007, 3, 255-260.

21. Astruc, D.; Lu, F.; Aranzaes, J.R. Nanoparticles as recyclable catalysts: The frontier between homogeneous and heterogeneous catalysis. Angew. Chem. Int. Ed. 2005, 44, 7852-7872.

22. Wang, D.; Astruc, D. Fast-growing field of magnetically recyclable nanocatalysts. Chem. Rev. 2014, 144, 6949-6985.

23. Zaera, F. Nanostructured materials for applications in heterogeneous catalysis. Chem. Soc. Rev. 2013, 42, 2746-2762. 
24. Joo, S.H.; Park, J.Y.; Tsung, C.K.; Yamasa, Y.; Yang, P.; Somorjai, G.A. Thermally stable $\mathrm{Pt} / \mathrm{mesoporous} \mathrm{silica} \mathrm{core-shell} \mathrm{nanocatalysts} \mathrm{for} \mathrm{high-temperature} \mathrm{reactions.} \mathrm{Nat.} \mathrm{Mater.} \mathrm{2009,} 8$, 126-131.

25. Lara, P.; Philippot, K. The hydrogenation of nitroarenes mediated by platinum nanoparticles: An overview. Catal. Sci. Technol. 2014, 4, 2445-2465.

26. Lu, Y.M.; Zhu, H.Z.; Li, W.G.; Hu, B.; Yu, S.H. Size-controllable palladium nanoparticles immobilized on carbon nanospheres for nitroaromatic hydrogenation. J. Mater. Chem. A 2013, 1, 3783-3788.

27. Kuroda, K.; Ishida, T.; Haruta, M. Reduction of 4-nitrophenol to 4-aminophenol over Au nanoparticles deposited on PMMA. J. Mol. Catal. A 2009, 298, 7-11.

28. Koizumi, N.; Jiang, X.; Kugai, J.; Song, C. Effects of mesoporous silica supports and alkaline promoters on activity of $\mathrm{Pd}$ catalysts in $\mathrm{CO}_{2}$ hydrogenation for methanol synthesis. Catal. Today 2012, 194, 16-24.

29. Hagen, J. Industrial Catalysis: A Practical Approach; Wiley-VCH: Weinhein, Germnay, 2006.

30. Linares, N.; Silvestre-Albero, A.M.; Serrano, E.; Silvestre-Albero, J.; Garcia-Martinez, J. Mesoporous materials for clean energy technologies. Chem. Soc. Rev. 2014, 43, 7681-7717.

31. Opanasenko, M.; Stepnicka, P.; Cejka, J. Heterogeneous Pd catalysts supported on silica matrices. RSC Adv. 2014, 4, 65137-65162.

32. Polshettiwar, V.; Asefa, T. Nanocatalysis, Synthesis and Applications; John Wiley \& Sons, Inc.: Hoboken, NJ, USA, 2013.

33. Polshettiwar, V.; Luque, R.; Fihri, A.; Zhu, H.; Bouhrara, M.; Basset, J.M. Magnetically recoverable nanocatalysts. Chem. Rev. 2011, 111, 3036-3075.

34. Gawande, M.B.; Monga, Y.; Zboril, R.; Sharma, R.K. Silica-decorated magnetic nanocomposites for catalytic applications. Coord. Chem. Rev. 2015, 288, 118-143.

35. Gawande, M.B.; Branco, P.; Varma, R.S. Nano-magnetite $\left(\mathrm{Fe}_{3} \mathrm{O}_{4}\right)$ as a support for recyclable catalysts in the development of sustainable methodologies. Chem. Soc. Rev. 2013, 42, 3371-3393.

36. Gawande, M.B.; Luque, R.; Zboril, R. The rise of magnetically recyclable nanocatalysts. ChemCatChem 2014, 6, 3312-3313.

37. Shokouhimehr, M.; Piao, Y.; Kim, J.; Jang, Y.; Hyeon, T. A magnetically recyclable nanocomposite catalyst for olefin epoxidation. Angew. Chem. Int. Ed. 2007, 46, 7039-7043.

38. $\mathrm{Mu}, \mathrm{B}$; Wang, A. One-pot fabrication of multifunctional superparamagnetic attapulgite $/ \mathrm{Fe}_{3} \mathrm{O}_{4} /$ polyaniline nanocomposites served as an adsorbent and catalyst support. J. Mater. Chem. A 2015, 3, 281-289.

39. Gawande, M.B.; Shelke, S.; Zboril, R.; Warma, R.S. Microwave-assisted chemistry: Synthetic applications for rapid assembly of nanomaterials and organics. Acc. Chem. Res. 2014, 47, 1338-1348.

40. Nasir Baig, R.B.; Varma, R.S. Magnetically retrievable catalysts for organic synthesis. Chem. Commun. 2013, 49, 754-770.

41. Deraedt, C.; Wang, D.; Salmon, L.; Etienne, L.; Labrugere, C.; Ruiz, J.; Astruc, D. Robust, efficient, and recyclable catalysts from the impregnation of preformed dendrimers containing palladium nanoparticles on a magnetic support. ChemCatChem 2015, 7, 303-308. 
42. Zhang, L.; Li, P.; Li, H.; Wang, L. A recyclable magnetic nanoparticles supported palladium catalyst for the Hiyama reaction of aryltrialkoxysilanes with aryl halides. Catal. Sci. Technol. 2012, 2, 1859-1864.

43. Gawande, M.B.; Rathi, A.K.; Branco, P.S.; Nogueira, I.D.; Velhinho, A.; Shrikhande, J.J.; Indulkar, U.U.; Jayaram, R.V.; Ghumman, C.A.A.; Bundaleski, N.; et al. Regio- and chemoselective reduction of nitroarenes and carbonyl compounds over recyclable magnetic ferrite-nickel nanoparticles $\left(\mathrm{Fe}_{3} \mathrm{O}_{4}-\mathrm{Ni}\right)$ by using glycerol as a hydrogen source. Chem. Eur. J. 2012, $18,12628-12632$.

44. Gawande, M.B.; Rathi, A.K.; Tucek, J.; Safarova, K.; Bundaleski, N.; Teodoro, O.M.N.D.; Kviteck, L.; Varma, R.S.; Zboril, R. Magnetic gold nanocatalyst (nanocat-Fe-Au): Catalytic applications for the oxidative esterification and hydrogen transfer reactions. Green Chem. 2014, 16, 4137-4143.

45. Pelisson, C.H.; Denicourt-Nowicki, A.; Meriadec, C.; Greneche, J.M.; Roucoux, A. Magnetically recoverable palladium(0) nanocomposite catalyst for hydrogenation reactions in water. ChemCatChem 2015, 7, 309-315.

46. Balanta, A.; Godard, C.; Claver, C. Pd nanoparticles for C-C coupling reactions. Chem. Soc. Rev. 2011, 40, 4973-4985.

47. Wang, Y.; Biradar, A.V.; Duncan, C.T.; Asefa, T. Silica nanosphere-supported shaped Pd nanoparticles encapsulated with nanoporous silica shell: Efficient and recyclable nanocatalysts. J. Mater. Chem. 2010, 20, 7834-7841.

48. Polshettiwar, V.; Cha, D.; Zhang, X.; Basset, J.M. High-surface-area silica nanospheres (KCC-1) with a fibrous morphology. Angew. Chem. Int. Ed. 2010, 49, 9652-9656.

49. Dong, Z.; Le, X.; Li, X.; Zhang, W.; Dong, C.; Ma, J. Silver nanoparticles immobilized on fibrous nano-silica as highlyefficient and recyclable heterogeneous catalyst for reduction of 4-nitrophenol and 2-nitroaniline. Appl. Catal. B 2014, 158, 129-135.

50. Johnson, J.A.; Makis, J.J.; Marvin, K.A.; Rodenbusch, S.E.; Stevenson, K. Size-dependent hydrogenation of $p$-nitrophenol with $\mathrm{Pd}$ nanoparticles synthesized with poly(amido)amine dendrimer templates. J. Phys. Chem. C 2013, 117, 22644-22651.

51. Dong, Z.; Le, X.; Dong, C.; Zhang, W.; Li, X.; Ma, J. Ni@Pd core-shell nanoparticles modified fibrous silica nanospheres as highly efficient and recoverable catalyst for reduction of 4-nitrophenol and hydrodechlorination of 4-chlorophenol. Appl. Catal. B 2015, 162, 372-380.

52. Metin, O.; Ho, S.F.; Alp, C.; Can, H.; Mankin, M.N.; Gultekin, S.; Chi, M.; Sun, S. Ni/Pd core/shell nanoparticles supported on graphene as a highly active and reusable catalyst for Suzuki-Miyaura cross-coupling reaction. Nano Res. 2013, 6, 10-18.

53. Kim, A.; Abdolhosseini, S.; Rafiaei, S.M.; Shokouhimehr, M. Palladium nanocatalysts confined in mesoporous silica for heterogeneous reduction of nitroaromatics. Energy Environ. Focus 2015, 4 , 18-23.

54. Thomas, J.M.; Johnson, B.F.G.; Raja, R.; Sankar, G.; Midgley, P.A. High-performance nanocatalysts for single-step hydrogenations. Acc. Chem. Res. 2003, 36, 20-30.

55. Park, S.; Lee, I.S.; Park, J. A magnetically separable gold catalyst for chemoselective reduction of nitro compounds. Org. Biomol. Chem. 2013, 11, 395-399. 
56. Jang, Y.; Kim, S.; Jun, S.W.; Kim, B.H.; Hwang, S.; Song, I.K.; Kim, B.M.; Hyeon, T. Simple one-pot synthesis of $\mathrm{Rh}-\mathrm{Fe}_{3} \mathrm{O}_{4}$ heterodimer nanocrystals and their applications to a magnetically recyclable catalyst for efficient and selective reduction of nitroarenes and alkenes. Chem. Commun. 2011, 47, 3601-3603.

57. Zhu, M.; Diao, G. Review on the progress in synthesis and application of magnetic carbon nanocomposites. Nanoscale 2011, 3, 2748-2767.

58. Zhu, Y.; Stubbs, L.P.; Ho, F.; Liu, R.; Ship, C.P.; Maguire, J.A.; Hosmane, N.S. Magnetic Nanocomposites: A new perspective in catalysis. ChemCatChem 2010, 2, 365-374.

59. Shokouhimehr, M.; Kim, T.; Jun, S.W.; Shin, K.; Jang, Y.; Kim, B.H.; Kim, J.; Hyeon, T. Magnetically separable carbon nanocomposite catalysts for efficientnitroarene reduction and Suzuki reactions. Appl. Catal. A 2014. 476, 133-139.

60. Li, H.; Zhu, Z.; Liu, J.; Xie, S.; Li, H. Hollow palladium-cobalt bimetallic nanospheres as an efficient and reusable catalyst for Sonogashira-type reactions. J. Mater. Chem. 2010, 20, 4366-4370.

61. Guan, Z.; Hu, J.; Gu, Y.; Zhang, H.; Li, G.; Li, T. PdCl 2 (py)2 encaged in monodispersed zeolitic hollow spheres: A highly efficient and reusable catalyst for Suzuki-Miyaura cross-coupling reaction in aqueous media. Green Chem. 2012, 14, 1964-1970.

62. Wang, P.; Zhu, H.; Liu, M.; Niu, J.; Yuan, B.; Li, R.; Ma, J. Stabilizing Pd on the surface of amine functionalized hollow $\mathrm{Fe}_{3} \mathrm{O}_{4}$ spheres: A highly active and recyclable catalyst for Suzuki cross coupling and hydrogenation reactions. RSC Adv. 2014, 4, 28922-28927.

63. Shokouhimehr, M.; Lee, J.E.; Han, S.I.; Hyeon, T. Magnetically recyclable hollow nanocomposite catalysts for heterogeneous reduction of nitroarenes and Suzuki reactions. Chem. Commun. 2013, 49, 4779-4781.

64. Yang, X.F.; Wang, A.; Qiao, B.; Li, J.; Liu, J.; Zhang, T. Single-atom catalysts: A new frontier in heterogeneous catalysis. Acc. Chem. Res. 2013, 46, 1740-1748.

65. Zhang, Q.; Lee, I.; Joo, J.B.; Zaera, F.; Yin, Y. Core-shell nanostructured catalysts. Acc. Chem. Res. 2013, 46, 1816-1824.

66. Xie, M.; Zhang, F.; Long, Y.; Ma, J. Pt nanoparticles supported on carbon coated magnetic microparticles: An efficient recyclable catalyst for hydrogenation of aromatic nitro-compounds. RSC. Adv. 2013, 3, 10329-10334.

67. Gawande, M.B.; Guo, H.; Rathi, A.K.; Branco, P.S.; Chen, Y.; Varma, R.S.; Peng, D.L. First application of core-shell Ag@Ni magnetic nanocatalyst for transfer hydrogenation reactions of aromatic nitro and carbonyl compounds. RSC Adv. 2013, 3, 1050-1054.

68. Choi, K.H.; Shokouhimehr, M.; Sung, Y.E. Heterogeneous Suzuki cross-coupling reaction catalyzed by magnetically recyclable nanocatalyst. Bull. Korean Chem. Soc. 2013, 34, 1477-1480.

69. Shokouhimehr, M.; Shin, K.Y.; Lee, J.S.; Hackett, M.J.; Jun, S.W.; Oh, M.H.; Jang, J.; Hyeon, T. Magnetically recyclable core-shell nanocatalysts for efficient heterogeneous oxidation of alcohols. J. Mater. Chem. A 2014, 2, 7593-7599.

70. Lynch, J.; Zhuang, J.; Wang, T.; LaMontagne, D.; W, H.; Cao, C. Gas-bubble effects on the formation of colloidal iron oxide nanocrystals. J. Am. Chem. Soc. 2011, 133, 12664-12674. 
71. Zeng, T.; Zhang, X.; Wang, S.; Ma, Y.; Niu, H.; Cai, Y. A double-shelled yolk-like structure as an ideal magnetic support of tiny gold nanoparticles for nitrophenol reduction. J. Mater. Chem. A 2013, $1,11641-11647$.

72. Yao, T.; Cui, T.; Fang, X.; Cui, F.; Wu, J. Preparation of yolk-shell Fe $\mathrm{O}_{y} / \mathrm{Pd} @$ mesoporous $\mathrm{SiO}_{2}$ composites with high stability and their application in catalytic reduction of 4-nitrophenol. Nanoscale 2013, 5, 596-6904.

73. Zhu, M.; Diao, G. Synthesis of porous $\mathrm{Fe}_{3} \mathrm{O}_{4}$ nanospheres and its application for the catalytic degradation of xylenol orange. J. Phys. Chem. C 2011, 115, 18923-18934.

(C) 2015 by the authors; licensee MDPI, Basel, Switzerland. This article is an open access article distributed under the terms and conditions of the Creative Commons Attribution license (http://creativecommons.org/licenses/by/4.0/). 